\title{
Rare earth element characteristics of pyrope garnets from the Kaavi-Kuopio kimberlites - implications for mantle metasomatism
}

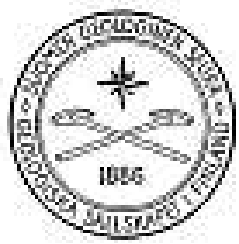

\author{
MarjaleEna Lehtonen \\ Geological Survey of Finland, P.O. Box 96, FIN-02151 Espoo, Finland
}

\begin{abstract}
Peridotitic garnet xenocrysts from five kimberlite pipes in the Kaavi-Kuopio area of eastern Finland have been studied using major and trace element geochemistry to obtain information on the stratigraphy, compositional variability and evolutionary history of the underlying lithospheric mantle. Ni thermometry on garnet xenocrysts gives $650-1350^{\circ} \mathrm{C}$ and, when extrapolated to the geotherm determined using mantle xenoliths, indicates a sampling interval of c. 80-230 km. Three distinct mantle layers are recognized based on the xenolith/xenocryst record: (I) A shallow, $<110 \mathrm{~km}$, garnet-spinel peridotite layer characterized by distinctive "CCGE" pyropes. (2) A variably depleted Iherzolitic and harzburgitic horizon from 110 to $180 \mathrm{~km}$. (3) A deep layer, $>180 \mathrm{~km}$, composed largely of fertile material. The chondrite-normalized REE profiles of subcalcic harzburgitic garnet xenocrysts originating from layer 2 bear evidence of an extensive ancient melt extraction event, similar to that observed in lithosphere underlying Archean cratons elsewhere. Memory of this event has possibly also been preserved in the $\mathrm{REE}_{N}$ signatures of rare depleted garnets from layer 3 and in the CCGE pyropes from layer I despite their saturation in Ca. The Iherzolitic and megacryst garnet varieties exhibit $L R E E_{N}$ depletion relative to $M R E E_{N}$ and $H R E E_{N}$, with the steady enrichment from $S m_{N}$ to $\mathrm{Yb}_{N}$ typical of Ca-saturated mantle garnets. The enrichment of MREE and HREE probably derives from a metasomatic event caused by silicate melts close in composition to megacryst magma, which also imprinted a Ti-metasomatic overprint on many pyrope garnets. Harzburgitic and rare lherzolitic garnets, however, appear to have escaped this metasomatism.
\end{abstract}

Key words: mantle, kimberlite, garnet group, xenocrysts, peridotites, xenoliths, geochemistry, rare earths, geologic thermometry, Kaavi, Kuopio, Finland 


\section{Introduction}

The Kaavi-Kuopio Kimberlite Province situated at the Karelian craton margin contains at least nineteen kimberlites with mineralogy typical of Group I kimberlite (Tyni, 1997; O’Brien \& Tyni, 1999). The pipes have intruded Archean (3.5-2.6 Ga) basement gneisses and allochthonous Proterozoic (1.9-1.8 Ga) metasediments thrust onto the craton during the Svecofennian orogeny (Kontinen et al., 1992; Fig. 1). Several methods have been used to date the kimberlite magmatism (Tyni, 1997; Peltonen et al., 1999; Peltonen \& Mänttäri, 2001) but the U-Pb ion probe ages of 589-626 Ma from perovskites (O'Brien et al., 2005) are considered to be the most reliable.
Mantle xenoliths and xenocrysts from the KaaviKuopio pipes have been studied by Peltonen (1999), Peltonen et al. (1999), Woodland \& Peltonen (1999) and Lehtonen et al. (2004). A geotherm corresponding to a $36 \mathrm{~mW} / \mathrm{m}^{2}$ has been calculated using heat flow constraints and xenolith modes, thermobarometry and thermal properties (Kukkonen \& Peltonen, 1999; Kukkonen et al., 2003). The xenolith/xenocryst suite provides evidence of vertical compositional variability of the lithospheric mantle adjacent to the ancient suture zone between the Archean Karelian craton and the Proterozoic Svecofennian mobile belt, with at least three distinct mantle horizons identified

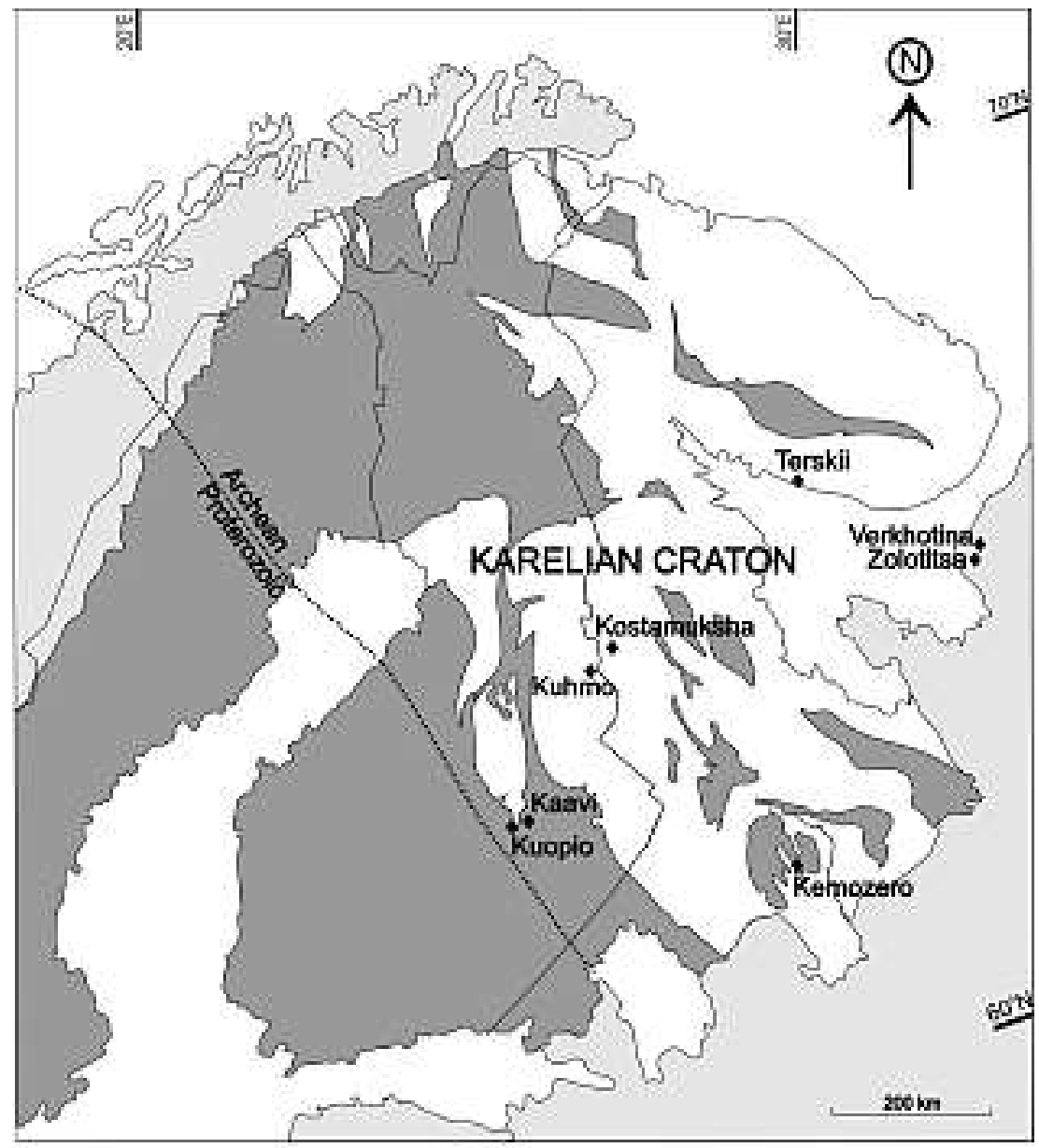

Avthean
Fig. I. Generalized geological map of Fennoscandia. The Archean/Proterozoic boundary marks the subsurface extent of the Archean craton as determined by $\mathrm{Nd}$ isotopes. The black diamonds represent diamond-bearing kimberlites and lamproites. 
(Lehtonen et al., 2004): 1. An upper zone, shallower than $110 \mathrm{~km}$, distinguished by Ca-saturated but Ti-, Y- and Zr-depleted pyropes. The rare xenoliths originating from this layer are garnet-spinel harzburgites, containing secondary Cpx. 2. A garnet peridotite zone occurs at 110 to $180 \mathrm{~km}$ depth, and ranges in bulk composition from harzburgite to lherzolite to wehrlite. 3. A deep, more fertile zone, from 180 to $240 \mathrm{~km}$, from where the bulk of the garnet facies peridotite xenoliths were derived. Mantle eclogite xenoliths originate also from this deepest layer, some of them being highly diamondiferous (Peltonen et al., 2002). The stabilization ages of these distinct manthe layers are still to be resolved but at least the upper and the middle layers are believed to be Archean, with a similar age to that of the overlying crust (Pearson, 1999). The lowermost layer may represent Archean lithospheric mantle that has been considerably re-fertilized by melt metasomatism (Lehtonen et al., 2004). Alternatively it is possible that this section of the mantle has been tectonically emplaced during the Svecofennian collision event at $1.88 \mathrm{Ga}$ (Peltonen et al., 1999). Re-Os isotope measurements on mantle xenoliths will eventually provide direct evidence on the stabilization ages of the different mantle horizons (Peltonen \& Brügmann, submitted).

The aim of this study was to obtain additional information on the composition, origin and evolutionary history of the stratified lithospheric mantle underlying Kaavi-Kuopio by studying major, trace, and rare-earth element contents of peridotitic garnet xenocrysts from five kimberlite pipes. Garnet xenocrysts do not contain as much information as mantle xenoliths because coexisting minerals are not available, but they are far more abundant in kimberlites and in principle provide a statistically robust sample of the underlying mantle (Schulze, 1995). The trace element contents of garnets and the shapes of their rare-earth element (REE) patterns normalized to C1chondrite composition, record a diverse range of geochemical behavior controlled by pressure, temperature and composition of their host rock, which themselves are related to the origin, provenance and thermal history of the mantle (e.g. Griffin et al., 1999a, 1999b; Canil et al., 2003). Garnet is a sensitive petrogenetic indicator, since it has very low $\mathrm{D}$ values for the light REE and increasingly larger D's for the heavy REE (Green, 1980), and provides information about depletion and metasomatic events prevalent in the lithospheric mantle prior to, or contemporaneous with, the kimberlite magmatism.

\section{Samples}

Samples were selected from five Kaavi-Kuopio Province kimberlites, Lahtojoki, Kylmälahti, Kärenpää, Niilonsuo and Ryönä (Table 1$)$. In the case of Lahtojoki and Kylmälahti kimberlites xenocryst grains $(0.25-2.0 \mathrm{~mm})$ were liberated by lightly crushing the kimberlite material followed by heavy medium separation and hand picking. The hard magmatic Niilonsuo kimberlite was processed in a similar way but first fragmented electrodynamically at Forschungszentrum Karlsruhe GmbH. The Kärenpää and Ryönä samples were heavy mineral concentrates handed over to the Geological Survey of Finland (GTK) by Malmikaivos Oy (Tyni, 1997).

Thousands of garnet xenocrysts were recovered from the kimberlite concentrates out of which c. 1300 were selected for analysis (Table 1). In this study an unsorted population of xenocrysts has been used in order to get unbiased information on the garnets present within the kimberlites. Except for the garnet-poor Niilonsuo kimberlite, the number of analyzed garnets per kimberlite does not represent (even though loosely correlates with) the total number of garnets within the sample.

\section{Analytical techniques}

Garnet major element compositions were determined by a Cameca Camebax SX50 electron microprobe (EMP) at GTK, applying an acceleration voltage of $25 \mathrm{kV}$, probe current of $48 \mathrm{nA}$ and beam diameter of $1 \mu \mathrm{m}$. Selected garnet xenocrysts were analyzed for trace elements by LA-ICP-MS at the University of Cape Town using the methodology and equipment described in Grégoire et al. (2003). Typical theoretical detection limits are in the range of $10-20 \mathrm{ppb}$ 
Table I. Description of kimberlite samples and the number of garnets selected for major and trace element analysis.

\begin{tabular}{l|c|l|l|l|c|c|c|c}
\hline Kimberlite & $\begin{array}{l}\text { Pipe } \\
\text { number }\end{array}$ & Location & Size & Sample type & $\begin{array}{l}\text { Sample } \\
\text { size }\end{array}$ & $\begin{array}{l}\text { Garnet } \\
\text { majors }\end{array}$ & $\begin{array}{l}\text { Garnet } \\
\text { traces* }\end{array}$ & $\begin{array}{l}\text { Garnet } \\
\text { REE }\end{array}$ \\
\hline Lahtojoki & 7 & Kaavi & 2 ha & weathered kimberlite material & $-4 \mathrm{~kg}$ & 614 & 192 & 73 \\
Kylmälahti & 17 & Kuopio & $2 \mathrm{ha}$ & drill core & $-1 \mathrm{~kg}$ & 188 & 102 & 57 \\
Kärenpää & 5 & Kaavi & $700 \times 30 \mathrm{~m}$ & concentrate & $-100 \mathrm{~g}$ & 95 & 12 & 9 \\
Niilonsuo & 2 & Kaavi & $300 \times 50 \mathrm{~m}$ & kimberlite boulders & $-2 \mathrm{~kg}$ & 18 & 9 \\
Ryönä & 10 & Kuopio & $2 \mathrm{ha}$ & concentrate & $-100 \mathrm{~g}$ & 392 & 186 & \\
\hline Total & & & & & & 1307 & 501 & 130 \\
\hline
\end{tabular}

*) $\mathrm{Ni}, \pm \mathrm{Y}, \pm \mathrm{Zr}, \pm \mathrm{Ga}, \pm \mathrm{Sc}$

for REE, Zr and Y, and 2 ppm for Ti and Ni. Trace $\mathrm{Ni}$ data by EMP were obtained on pyrope grains employing $500 \mathrm{nA}$ probe current, $600 \mathrm{~s}$ counting times on peak plus background positions and were reduced by the CSIRO TRACE program for the SX50 (Robinson \& Graham, 1992). Cross-checking of the trace methods shows that $\mathrm{Ni}$ analyses in pyrope by EMP can achieve similar precision to those of LA ICP-MS down to the level of ca. $10 \mathrm{ppm}$.

The garnets were classified based on their major element composition into harzburgitic, lherzolitic, wehrlitic, megacrystal, eclogitic and crustal varieties according to Schulze (2003). In addition, the term "CCGE" which stands for "chromite-clinopyroxene-garnet equilibrium" was adopted from Kopylova et al. (2000) for distinctive Ca-saturated garnets, which in this study have low Ti, Y and Zr. For equilibration temperatures the Ni thermometer (Griffin et al., 1989a) was applied using the calibration of Ryan et al. (1996).

\section{Review of previous results}

Major part of the following results of garnet geochemistry and Ni thermometry from Kaavi-Kuopio kimberlites has been discussed already in Lehtonen et al. (2004). However, this study also contains major and trace element data on garnets from Ryönä kimberlite (Table 1), which was not included in the previous study. Selected major and trace element analyses for pyrope xenocrysts are presented in Table 2. The entire analytical database is available as an Open file at the Geological Survey of Finland (Lehtonen et al.,
2005) or can be requested from the author by e-mail. Major and trace element analyses from garnets in the Kaavi-Kuopio mantle xenoliths have been published in Peltonen et al. (1999).

\section{I. Garnet major element geochemistry}

$\mathrm{CaO}$ and $\mathrm{Cr}_{2} \mathrm{O}_{3}$ contents of garnets from Kaavi-Kuopio peridotite xenoliths and garnet xenocrysts are shown in Figure 2 and the classification of xenocrysts based on Schulze (2003) is presented in Figure 3. It is evident that lherzolitic garnet predominates over other garnet varieties. Moreover, a well-developed pyrope trend (CCGE) exists similar to that described by Kopylova et al. (1999; 2000) and Carbno \& Canil (2002) from the Slave craton (Fig. 2). Orange relatively $\mathrm{Cr}$-poor but $\mathrm{Ti}$-rich pyropes of megacryst composition are also common among the xenocrysts. A few grains in the lower right corner of the $\mathrm{CaO}-\mathrm{Cr}_{2} \mathrm{O}_{3}$ diagram, at high levels of $\mathrm{Ca}$ and low levels of Cr, fall into the "eclogitic" category (Schulze, 2003). None of the garnets from xenoliths and only a minor portion of the garnet xenocrysts are classified as subcalcic harzburgitic grains.

\subsection{Garnet thermometry and trace element geochemistry}

The $\mathrm{T}_{\mathrm{Ni}}$ histogram in Figure 4 for Kaavi-Kuopio garnet xenocrysts shows bimodal distribution including a strong low temperature peak at 700 to $850^{\circ} \mathrm{C}$ consisting dominantly of CCGE pyropes and a stronger 


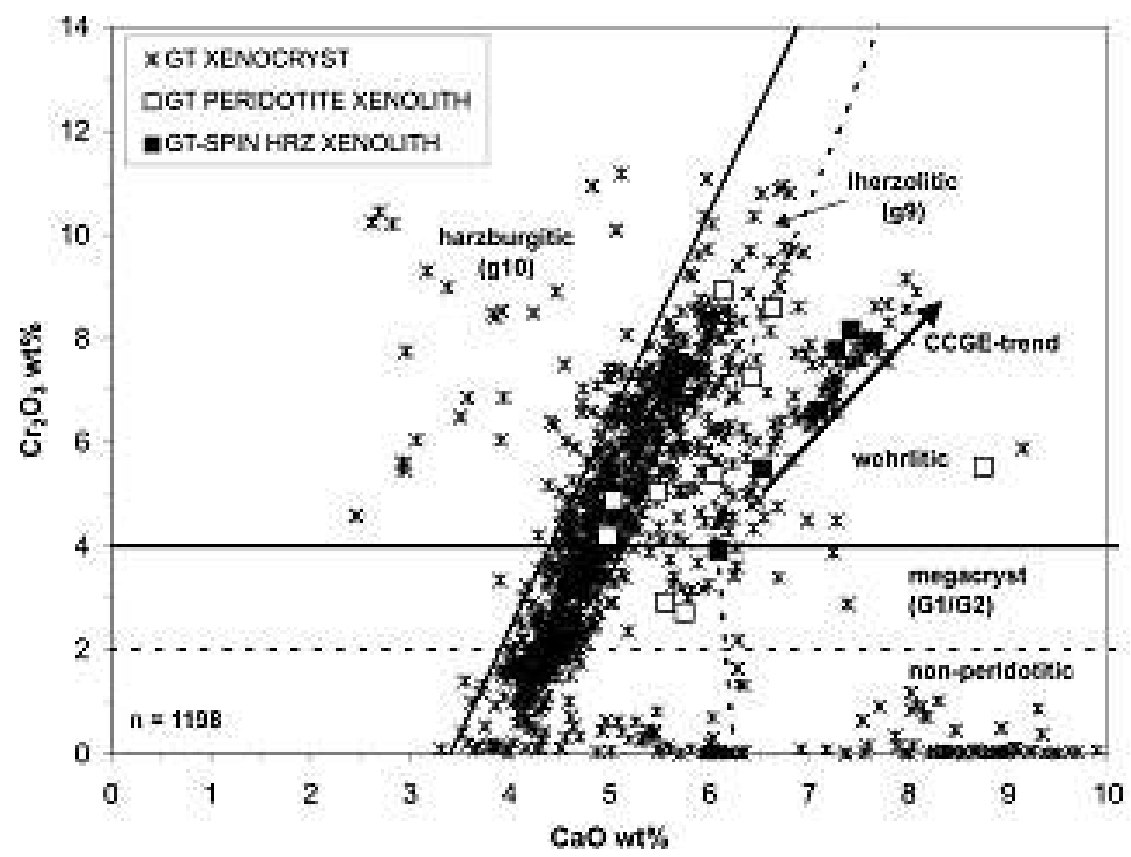

sampling peak at 1000 to $1150^{\circ} \mathrm{C}$ consisting of lherzolitic, harzburgitic and megacryst varieties. CCGE pyropes do not occur at higher temperatures than $850^{\circ} \mathrm{C}$. Based on the $\mathrm{Y}$ contents of garnets, $1150^{\circ} \mathrm{C}$ represents another break in the lithosphere, separating mantle that contains strongly depleted pyropes from more enriched mantle, as seen in Figure 5 where $\mathrm{T}_{\mathrm{Ni}}$ temperatures have been extrapolated to the local geotherm (Kukkonen and Peltonen, 1999; Kukkonen et al., 2003). The "Y edge" (terminology after Griffin \& Ryan, 1995) does not mark the lower boundary of the lithosphere, as all xenoliths from $>1150^{\circ} \mathrm{C}$ are coarse granular peridotites typical of subcontinental lithospheric mantle (Peltonen et al., 1999). Figure 5 shows that the main horizon of sampling at 1000 to $1150^{\circ} \mathrm{C}$ corresponds to the mantle section from 140 to $175 \mathrm{~km}$.

The two breaks, 850 and $1150^{\circ} \mathrm{C}$, in the mantle stratigraphy show up as well using the $\mathrm{TiO}_{2}$ contents and $\mathrm{Mg \#}$ of garnets (Fig. 6). Variations in the pyrope $\mathrm{TiO}_{2}$ contents as a function of temperature closely follow the same pattern as the pyrope $\mathrm{Y}$ contents, since both of these elements reflect the degree of depletion or enrichment within the mantle (e.g. Griffin \& Ryan, 1995). Mg\# is a substantially different parameter of garnet composition than Ti and Y. Mg\# of
Fig. 2. $\mathrm{Cr}_{2} \mathrm{O}_{3}$ vs. $\mathrm{CaO}$ of Kaavi-Kuopio kimberlite-derived garnets. The xenocryst data are representative of the entire mantle garnet population of the pipes. Crustal garnets are excluded from this diagram using the classfication by Schulze (2003). The harzburgite, Iherzolite and non-peridotite fields are redrawn after Gurney (1984) and the wehrlite field is separated according to Sobolev et al. (1973). Arrow pointing NE marks the "CCGE" garnet trend, i.e. chromite-clinopyroxene-garnet equilibrium, recognized in spinel-garnet peridotites from the Jericho kimberlite (Kopylova et al., 2000).

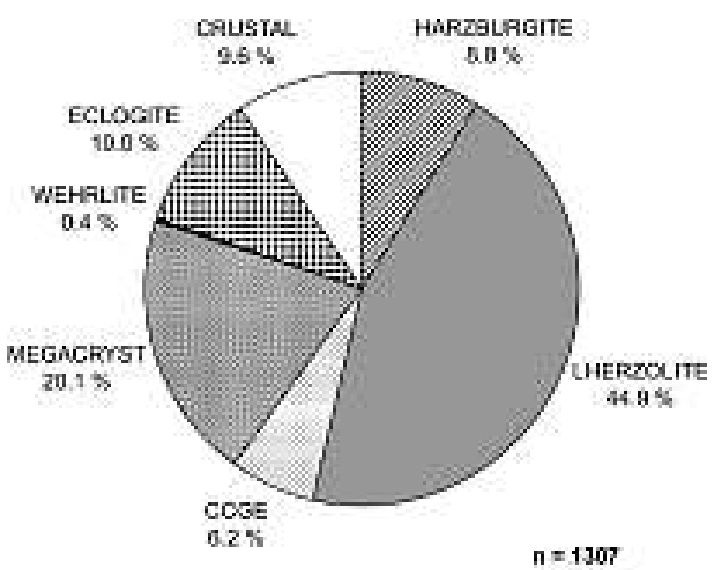

Fig. 3. Classification of garnet xenocrysts recovered from heavy mineral concentrates according to the scheme of Schulze (2003). Term "CCGE” adopted from Kopylova et al. (2000) for distinct garnets (discussed in text); using the Schulze classification these garnets would be labelled as Iherzolitic varieties. 
Table 2. Representative*) electron microprobe and LA-ICP-MS analyses of garnet xenocrysts from Finnish kimberlites.

\begin{tabular}{|c|c|c|c|c|c|c|c|c|c|c|c|c|c|}
\hline & & & EPM & analys & & & & & & & & & \\
\hline 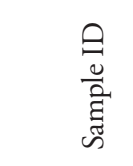 & 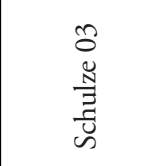 & 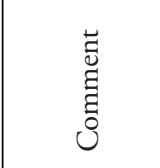 & 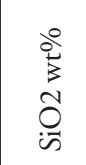 & 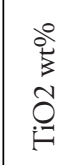 & 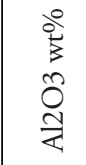 & 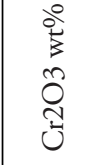 & 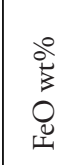 & $\begin{array}{l}0^{0} \\
0^{0} \\
\sum^{5}\end{array}$ & $\begin{array}{l}\sum_{0}^{0} \\
0_{0}^{\infty} \\
\sum^{2}\end{array}$ & 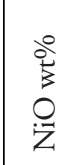 & 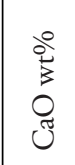 & 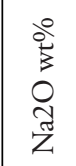 & $\frac{\stackrel{0}{3}}{\frac{\sum^{\circ}}{0}}$ \\
\hline 4682.02 & Lherzolite & CCGE & 39.61 & 0.05 & 20.21 & 5.01 & 8.74 & 0.48 & 18.35 & 0.00 & 6.38 & 0.00 & 98.92 \\
\hline 10657.02 & Lherzolite & CCGE & 40.17 & 0.00 & 17.68 & 7.55 & 8.25 & 0.53 & 17.16 & 0.00 & 7.44 & 0.00 & 98.83 \\
\hline 4398.96 & Lherzolite & CCGE & 40.83 & 0.00 & 17.01 & 8.62 & 7.74 & 0.56 & 17.60 & 0.00 & 7.67 & n.a. & 100.08 \\
\hline 10000.02 & Lherzolite & CCGE & 40.16 & 0.01 & 17.90 & 7.55 & 8.20 & 0.51 & 17.60 & 0.00 & 7.43 & 0.01 & 99.40 \\
\hline$\# 9$ & Lherzolite & Sympl. type & 40.18 & 0.04 & 17.83 & 7.39 & 7.85 & 0.55 & 19.07 & 0.00 & 5.54 & n.a. & 98.44 \\
\hline \#31 & Harzburgite & Sympl. type & 40.94 & 0.02 & 18.60 & 6.58 & 8.34 & 0.61 & 19.65 & 0.00 & 4.72 & n.a. & 99.47 \\
\hline 8101.96 & Lherzolite & Sympl. type & 40.65 & 0.24 & 14.50 & 10.87 & 6.62 & 0.39 & 19.34 & 0.01 & 6.70 & n.a. & 99.48 \\
\hline 8103.96 & Lherzolite & Sympl. type & 40.62 & 0.27 & 14.41 & 10.94 & 6.58 & 0.41 & 19.24 & 0.02 & 6.74 & n.a. & 99.35 \\
\hline 4403.96 & Harzburgite & Sinusoidal & 42.19 & 0.03 & 19.35 & 6.05 & 6.44 & 0.36 & 23.19 & 0.01 & 3.06 & n.a. & 100.69 \\
\hline$\# 43$ & Harzburgite & Sinusoidal & 41.00 & 0.28 & 17.24 & 8.08 & 6.22 & 0.32 & 20.81 & 0.01 & 5.18 & n.a. & 99.16 \\
\hline 15947.01 & Harzburgite & Sinusoidal & 41.05 & 0.11 & 16.81 & 8.49 & 6.31 & 0.32 & 21.26 & 0.01 & 4.24 & n.a. & 98.68 \\
\hline 15946.01 & Harzburgite & Sinusoidal & 41.34 & 0.31 & 18.08 & 6.59 & 6.49 & 0.29 & 21.04 & 0.01 & 4.83 & n.a. & 99.00 \\
\hline 15971.01 & Harzburgite & Sinusoidal & 40.89 & 0.07 & 15.90 & 10.21 & 6.31 & 0.36 & 22.35 & 0.02 & 2.82 & n.a. & 98.95 \\
\hline 18078.01 & Lherzolite & Sinusoidal & 41.16 & 0.34 & 17.46 & 7.57 & 7.03 & 0.41 & 20.26 & 0.01 & 5.56 & n.a. & 99.82 \\
\hline 18092.01 & Lherzolite & Sinusoidal & 40.87 & 0.25 & 16.93 & 8.39 & 6.90 & 0.38 & 19.81 & 0.01 & 6.05 & n.a. & 99.58 \\
\hline 13374.95 & Lherzolite & Sinusoidal & 41.05 & 0.36 & 16.64 & 8.56 & 6.65 & 0.40 & 20.04 & 0.01 & 6.05 & n.a. & 99.91 \\
\hline 4686.02 & Lherzolite & Sinusoidal & 40.18 & 0.03 & 18.57 & 5.90 & 6.80 & 0.26 & 20.95 & 0.02 & 5.78 & 0.01 & 98.65 \\
\hline 15995.01 & Wehrlite & N-type & 41.03 & 0.58 & 19.20 & 4.49 & 9.93 & 0.51 & 17.29 & 0.01 & 7.28 & n.a. & 100.33 \\
\hline \#106 & Lherzolite & $\mathrm{N}$-type & 40.04 & 0.53 & 17.31 & 6.89 & 8.98 & 0.53 & 17.58 & 0.01 & 6.85 & n.a. & 98.76 \\
\hline \#94 & Lherzolite & $\mathrm{N}$-type & 39.99 & 0.91 & 15.99 & 7.71 & 9.27 & 0.42 & 17.64 & 0.01 & 6.87 & n.a. & 98.82 \\
\hline 15924.01 & Lherzolite & N-type & 40.14 & 0.88 & 17.62 & 5.70 & 9.35 & 0.42 & 17.84 & 0.01 & 6.37 & n.a. & 98.38 \\
\hline 10660.02 & Lherzolite & $\mathrm{N}$-type & 40.21 & 0.90 & 16.82 & 6.24 & 9.75 & 0.42 & 18.04 & 0.01 & 6.12 & 0.11 & 98.69 \\
\hline 4674.02 & Lherzolite & $\mathrm{N}$-type & 39.58 & 0.15 & 20.11 & 4.55 & 8.56 & 0.42 & 19.91 & 0.01 & 5.20 & 0.05 & 98.59 \\
\hline 10635.02 & Lherzolite & N-type & 40.69 & 0.54 & 19.37 & 4.08 & 8.52 & 0.40 & 19.39 & 0.01 & 5.55 & 0.09 & 98.69 \\
\hline 13358.95 & Lherzolite & $\mathrm{N}$-type & 41.13 & 0.55 & 19.75 & 4.35 & 7.05 & 0.41 & 20.80 & 0.01 & 4.83 & n.a. & 99.06 \\
\hline 4692.02 & Lherzolite & N-type & 39.72 & 0.63 & 19.71 & 4.18 & 8.48 & 0.41 & 19.63 & 0.01 & 5.40 & 0.07 & 98.33 \\
\hline 4611.02 & Lherzolite & N-type & 40.37 & 0.86 & 18.67 & 5.49 & 7.29 & 0.38 & 20.46 & 0.00 & 5.32 & 0.11 & 98.99 \\
\hline$\# 11$ & Lherzolite & $\mathrm{N}$-type & 40.36 & 0.61 & 16.74 & 7.86 & 8.20 & 0.48 & 19.04 & 0.00 & 5.97 & n.a. & 99.27 \\
\hline 8108.96 & Lherzolite & N-type & 42.41 & 0.47 & 19.92 & 3.73 & 6.71 & 0.31 & 22.14 & 0.02 & 4.72 & n.a. & 100.59 \\
\hline 18147.01 & Lherzolite & $\mathrm{N}$-type & 41.44 & 0.54 & 19.45 & 4.01 & 7.58 & 0.28 & 21.20 & 0.01 & 4.80 & n.a. & 99.42 \\
\hline 18155.01 & Lherzolite & N-type & 41.55 & 0.56 & 18.95 & 5.05 & 7.06 & 0.30 & 21.00 & 0.01 & 5.25 & n.a. & 99.76 \\
\hline 18077.01 & Lherzolite & N-type & 41.43 & 0.56 & 17.25 & 6.96 & 6.73 & 0.28 & 20.91 & 0.01 & 5.57 & n.a. & 99.75 \\
\hline 8109.96 & Lherzolite & N-type & 41.27 & 0.60 & 19.25 & 4.07 & 7.73 & 0.37 & 20.89 & 0.02 & 4.94 & n.a. & 99.30 \\
\hline 10634.02 & Lherzolite & $\mathrm{N}$-type & 40.33 & 0.89 & 17.28 & 6.28 & 8.45 & 0.44 & 19.02 & 0.01 & 6.04 & 0.09 & 98.86 \\
\hline$\# 17$ & Lherzolite & N-type & 41.68 & 0.41 & 20.88 & 2.39 & 7.14 & 0.30 & 21.69 & 0.01 & 4.63 & n.a. & 99.19 \\
\hline 4703.02 & Lherzolite & N-type & 40.93 & 0.49 & 21.04 & 2.40 & 8.23 & 0.33 & 21.58 & 0.02 & 4.40 & 0.06 & 99.56 \\
\hline 4669.02 & Lherzolite & $\mathrm{N}$-type & 41.06 & 0.49 & 21.11 & 2.01 & 8.18 & 0.29 & 21.19 & 0.01 & 4.70 & 0.05 & 99.14 \\
\hline \#22 & Lherzolite & $\mathrm{N}$-type & 40.89 & 0.03 & 19.54 & 5.09 & 8.49 & 0.55 & 19.11 & 0.00 & 5.72 & n.a. & 99.52 \\
\hline 13362.95 & Megacryst & N-type & 41.46 & 0.52 & 21.11 & 2.08 & 7.71 & 0.32 & 21.13 & 0.02 & 4.41 & n.a. & 98.91 \\
\hline 4655.02 & Megacryst & N-type & 40.50 & 0.64 & 21.05 & 2.16 & 8.03 & 0.28 & 21.31 & 0.01 & 4.76 & 0.04 & 98.88 \\
\hline 18160.01 & Megacryst & N-type & 41.28 & 0.65 & 20.57 & 2.51 & 8.33 & 0.29 & 20.90 & 0.01 & 4.85 & n.a. & 99.41 \\
\hline 4672.02 & Megacryst & N-type & 40.23 & 0.77 & 20.23 & 2.91 & 8.50 & 0.34 & 20.67 & 0.01 & 5.00 & 0.06 & 98.79 \\
\hline 8114.96 & Megacryst & N-type & 41.30 & 0.64 & 20.74 & 1.48 & 9.07 & 0.37 & 20.76 & 0.02 & 4.23 & n.a. & 98.79 \\
\hline
\end{tabular}

*) The entire analytical database is available as the Geological Survey of Finland Open file report M 41.2/2005/1 (Lehtonen et al., 2005) Schulze 03 = Schulze (2003); RGP96 = Ryan et al. (1996)

n.a. = not analyzed

n.d. = not detected 


\begin{tabular}{|c|c|c|c|c|c|c|c|c|c|c|c|c|c|c|c|}
\hline \multicolumn{15}{|c|}{ LA-ICP-MS analyses } & \multirow[b]{2}{*}{ 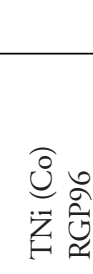 } \\
\hline 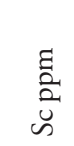 & 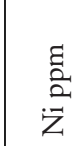 & 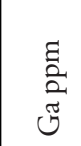 & 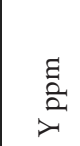 & 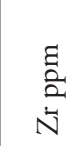 & $\begin{array}{l}\tilde{\Xi} \\
\tilde{a} \\
\widetilde{\Xi}\end{array}$ & 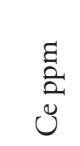 & $\begin{array}{l}\text { हă } \\
\text { à } \\
\vec{z} \\
\vec{z}\end{array}$ & $\begin{array}{l}\text { हू } \\
\text { a. } \\
\text { हn }\end{array}$ & $\begin{array}{l}\text { घี } \\
\text { م્ } \\
\vec{\Xi} \\
\text { 외 }\end{array}$ & 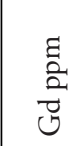 & $\begin{array}{l}\tilde{z} \\
\hat{a} \\
\hat{a}\end{array}$ & 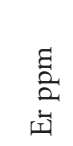 & $\begin{array}{l}\text { घี } \\
\text { ă } \\
\text { ह્ર }\end{array}$ & 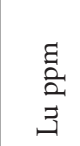 & \\
\hline 135 & 12 & 2.2 & 2.8 & 3.8 & 0.08 & 0.13 & 0.53 & 0.18 & 0.08 & 0.18 & 0.28 & 0.51 & 0.62 & 0.25 & 688 \\
\hline 208 & 20 & 2.8 & 0.2 & 0.8 & 0.09 & 0.16 & 0.13 & 0.17 & 0.08 & 0.09 & 0.11 & n.d. & 0.35 & 0.08 & 795 \\
\hline 284 & 23 & 2.5 & 0.5 & 2.4 & 0.13 & 1.01 & 1.06 & 0.17 & 0.03 & 0.18 & 0.16 & 0.01 & 0.34 & 0.14 & 826 \\
\hline 197 & 19 & 2.5 & 0.3 & 1.2 & 0.08 & 0.28 & 0.33 & 0.15 & 0.04 & 0.16 & 0.05 & 0.08 & 0.36 & 0.14 & 777 \\
\hline 220 & 21 & 2.4 & 17.3 & 44.6 & 0.03 & 0.85 & 5.63 & 3.48 & 1.35 & 4.49 & 3.70 & 1.39 & 1.32 & 0.28 & 803 \\
\hline 175 & 22 & 3.4 & 6.6 & 40.0 & 0.02 & 0.80 & 4.07 & 3.03 & 1.18 & 4.11 & 2.09 & 0.37 & 0.09 & 0.08 & 814 \\
\hline 128 & 71 & 9.0 & 19.9 & 41.2 & 0.22 & 1.21 & 6.39 & 5.41 & 1.90 & 7.19 & 4.76 & 1.28 & 1.02 & 0.23 & 1154 \\
\hline 131 & 71 & 8.9 & 20.2 & 43.9 & 0.20 & 1.07 & 7.23 & 5.75 & 2.11 & 6.73 & 4.77 & 1.76 & 0.74 & 0.18 & 1156 \\
\hline 113 & 50 & 3.1 & 1.1 & 3.9 & 0.12 & 0.36 & 2.95 & 0.82 & 0.19 & 0.57 & 0.17 & 0.18 & 0.11 & 0.11 & 1034 \\
\hline 119 & 62 & 11.3 & 3.2 & 15.8 & 0.12 & 0.46 & 1.53 & 0.63 & 0.20 & 0.70 & 0.57 & 0.37 & 0.70 & 0.15 & 1106 \\
\hline 171 & 65 & 5.1 & 3.3 & 5.7 & 0.07 & 0.78 & 2.94 & 1.06 & 0.27 & 0.98 & 0.51 & 0.42 & 0.48 & 0.07 & 1122 \\
\hline 130 & 66 & 5.5 & 3.4 & 21.4 & 0.08 & 0.29 & 1.07 & 0.92 & 0.27 & 1.07 & 0.89 & 0.54 & 0.70 & 0.06 & 1124 \\
\hline 158 & 67 & 2.9 & 1.0 & 18.8 & 0.08 & 0.54 & 3.06 & 2.04 & 0.46 & 1.39 & 0.32 & 0.06 & 0.24 & 0.07 & 1134 \\
\hline 147 & 55 & 3.8 & 4.4 & 38.0 & 0.08 & 0.37 & 1.44 & 1.07 & 0.32 & 1.67 & 1.17 & 0.38 & 0.47 & 0.14 & 1061 \\
\hline 140 & 53 & 3.4 & 1.0 & 13.3 & 0.12 & n.d. & 1.61 & 0.73 & 0.08 & 0.30 & 0.12 & 0.11 & 0.47 & 0.05 & 1047 \\
\hline 141 & 77 & 4.9 & 1.9 & 18.7 & 0.08 & 0.41 & 1.53 & 0.94 & 0.19 & 0.86 & 0.34 & 0.23 & 0.35 & 0.13 & 1185 \\
\hline 128 & 100 & 5.3 & 1.3 & 1.2 & 0.14 & 0.71 & 1.21 & 0.46 & 0.13 & 0.11 & 0.09 & 0.18 & 0.53 & 0.12 & 1297 \\
\hline 138 & 44 & 13.9 & 25.2 & 64.3 & 0.06 & 0.39 & 1.22 & 1.09 & 0.67 & 2.57 & 4.71 & 3.07 & 3.08 & 0.44 & 993 \\
\hline 141 & 46 & 9.1 & 22.5 & 63.9 & 0.10 & 0.43 & 1.46 & 1.03 & 0.56 & 2.95 & 4.17 & 2.36 & 2.18 & 0.35 & 1006 \\
\hline 118 & 57 & 13.9 & 17.5 & 50.2 & 0.12 & 0.35 & 1.05 & 0.78 & 0.40 & 2.01 & 2.97 & 2.24 & 1.95 & 0.30 & 1071 \\
\hline 123 & 59 & 14.9 & 17.0 & 46.2 & 0.12 & 0.52 & 1.41 & 0.87 & 0.46 & 1.64 & 3.07 & 2.27 & 2.36 & 0.41 & 1084 \\
\hline 115 & 64 & 15.2 & 16.8 & 49.0 & 0.11 & 0.33 & 0.93 & 0.74 & 0.46 & 2.20 & 3.02 & 1.83 & 1.74 & 0.25 & 1112 \\
\hline 124 & 34 & 4.6 & 13.1 & 46.5 & 0.06 & 0.45 & 1.84 & 1.32 & 0.53 & 2.02 & 2.25 & 1.39 & 1.27 & 0.24 & 918 \\
\hline 96 & 46 & 9.7 & 15.6 & 42.3 & 0.04 & 0.23 & 0.98 & 0.57 & 0.36 & 1.39 & 2.98 & 1.75 & 1.54 & 0.35 & 1004 \\
\hline 90 & 52 & 7.0 & 11.5 & 28.3 & 0.06 & 0.22 & 0.74 & 0.62 & 0.26 & 1.23 & 1.84 & 1.16 & 1.68 & 0.23 & 1041 \\
\hline 86 & 38 & 8.2 & 14.7 & 34.2 & 0.05 & 0.21 & 0.70 & 0.45 & 0.32 & 1.43 & 2.60 & 1.70 & 1.55 & 0.26 & 947 \\
\hline 110 & 63 & 9.2 & 14.2 & 44.6 & 0.04 & 0.29 & 0.90 & 0.79 & 0.43 & 1.58 & 2.55 & 1.52 & 1.77 & 0.24 & 1109 \\
\hline 123 & 40 & 5.7 & 14.0 & 43.4 & 0.04 & 0.33 & 1.60 & 1.08 & 0.46 & 1.83 & 2.54 & 1.21 & 1.11 & 0.19 & 964 \\
\hline 94 & 112 & 7.8 & 10.9 & 28.6 & 0.05 & 0.30 & 1.38 & 0.66 & 0.27 & 1.38 & 1.90 & 1.02 & 1.43 & 0.28 & 1355 \\
\hline 113 & 84 & 7.9 & 13.6 & 35.1 & 0.09 & 0.24 & 1.07 & 0.65 & 0.29 & 1.19 & 2.50 & 1.30 & 1.39 & 0.26 & 1222 \\
\hline 113 & 79 & 8.6 & 12.8 & 43.3 & 0.09 & 0.30 & 1.28 & 0.87 & 0.26 & 1.47 & 2.27 & 1.29 & 0.97 & 0.20 & 1196 \\
\hline 135 & 118 & 6.8 & 10.3 & 41.0 & 0.07 & 0.45 & 2.15 & 0.98 & 0.33 & 1.74 & 1.70 & 1.24 & 0.94 & 0.19 & 1383 \\
\hline 102 & 72 & 9.2 & 13.4 & 39.3 & 0.03 & 0.24 & 1.02 & 0.71 & 0.35 & 1.45 & 2.09 & 1.36 & 1.87 & 0.31 & 1160 \\
\hline 101 & 71 & 14.2 & 15.0 & 44.3 & 0.05 & 0.30 & 0.93 & 0.80 & 0.52 & 1.65 & 2.83 & 1.79 & 1.95 & 0.32 & 1155 \\
\hline 83 & 77 & 6.7 & 11.0 & 31.8 & 0.05 & 0.32 & 0.75 & 0.56 & 0.31 & 1.03 & 1.86 & 1.50 & 1.50 & 0.24 & 1188 \\
\hline 84 & 83 & 7.6 & 12.8 & 27.1 & 0.04 & 0.24 & 0.70 & 0.45 & 0.40 & 0.85 & 1.87 & 1.59 & 1.68 & 0.35 & 1216 \\
\hline 94 & 86 & 8.3 & 12.4 & 33.7 & 0.06 & 0.30 & 1.13 & 0.66 & 0.39 & 1.41 & 1.84 & 1.52 & 1.56 & 0.35 & 1234 \\
\hline 92 & 77 & 7.3 & 9.2 & 28.4 & 0.15 & 0.54 & 0.96 & 0.51 & 0.30 & 0.91 & 1.96 & 1.19 & 1.40 & 0.22 & 1185 \\
\hline 73 & 86 & 9.6 & 13.4 & 25.9 & 0.04 & 0.25 & 0.66 & 0.55 & 0.29 & 1.32 & 1.95 & 1.69 & 1.77 & 0.29 & 1233 \\
\hline 98 & 80 & 8.3 & 13.6 & 37.3 & 0.11 & 0.37 & 0.80 & 0.74 & 0.42 & 1.27 & 2.03 & 1.52 & 2.10 & 0.32 & 1202 \\
\hline 94 & 77 & 10.4 & 14.3 & 40.1 & 0.04 & 0.22 & 0.90 & 0.63 & 0.33 & 1.02 & 2.28 & 1.45 & 1.91 & 0.32 & 1187 \\
\hline 111 & 63 & 9.5 & 14.7 & 42.5 & 0.02 & 0.25 & 0.91 & 0.55 & 0.31 & 1.25 & 2.32 & 1.94 & 2.03 & 0.28 & 1108 \\
\hline 96 & 71 & 9.9 & 16.2 & 38.8 & 0.04 & 0.20 & 0.83 & 0.50 & 0.32 & 1.67 & 2.98 & 2.19 & 2.40 & 0.44 & 1155 \\
\hline
\end{tabular}




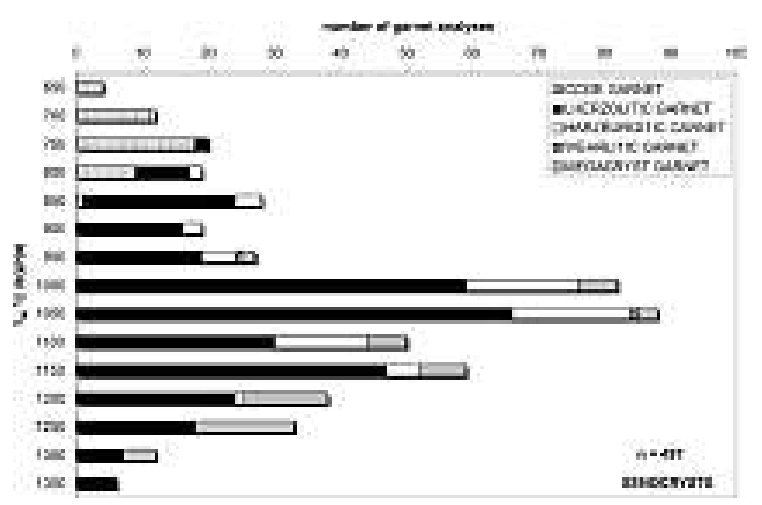

Fig. 4. Distribution of $\mathrm{T}_{\mathrm{Ni}}$ for Kaavi-Kuopio pyrope xenocrysts using the calibration of Ryan et al. (1996). Tirich megacryst composition garnets are included for illustrative purposes. Megacryst garnets with $\mathrm{Cr}_{2} \mathrm{O}_{3}>1.5$ wt\% and $\mathrm{MgO}>18 \mathrm{wt} \%$ are shown as they reach the same contents of these elements as do Iherzolitic pyropes, implying equilibration with olivine similar to that in the Iherzolites.

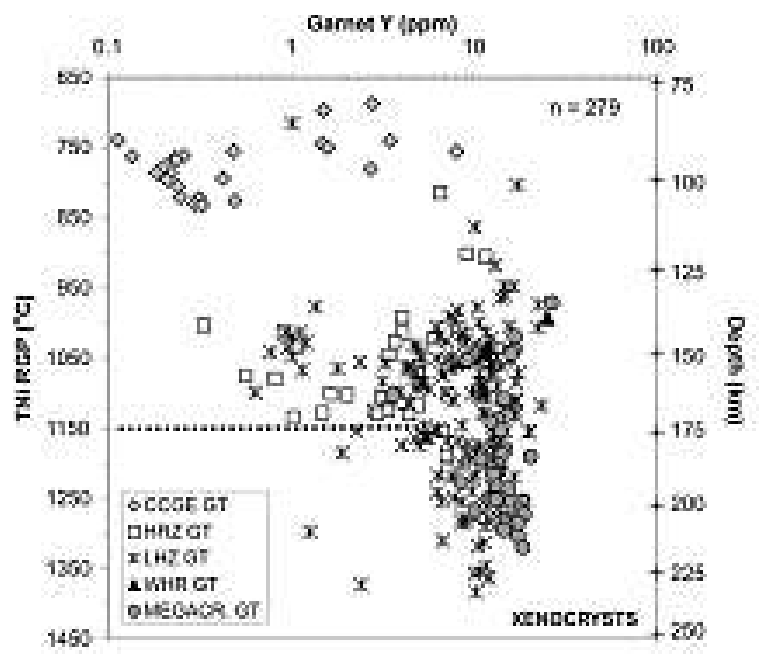

Fig. 5. $Y$ contents of pyropes vs. $T_{\mathrm{Ni}}$ (Ryan et al., 1996) show an edge at about $1150^{\circ} \mathrm{C}$ (modified from Lehtonen et al., 2004). Depths marked on the right vertical axis of the diagram are calculated by extrapolating the $\mathrm{T}_{\mathrm{Ni}}$ temperatures of pyropes to the local geotherm determined by Kukkonen et al. (2003). Cr-rich $\left(\mathrm{Cr}_{2} \mathrm{O}_{3}>\mathrm{I} .5\right.$ wt\%) megacryst composition grains are included in the diagrams, see Fig. Cap. 4.

garnet reflects bulk composition but it is also strongly controlled by the temperature dependent $\mathrm{Mg}-\mathrm{Fe}$ exchange with olivine, Opx, Cpx and Mg-chromite
(Stachel et al., 2003). Moreover, Ca has a significant crystal chemical effect on the $\mathrm{Mg}$-Fe partitioning between garnet and olivine (Stachel et al., 2003; O’Neill $\&$ Wood, 1979). The lower temperature boundary at c. $850^{\circ} \mathrm{C}$ corresponds to the break in $\mathrm{TiO}_{2}$ at approximately $110 \mathrm{~km}$ depth and separates extremely low Ti content CCGE pyropes with $\mathrm{Mg} \#$ around 0.80 from a lherzolitic and harzburgitic (and wehrlitic) horizon exhibiting a wide range in both parameters. The second boundary roughly at $1150^{\circ} \mathrm{C}$ or ca. $175 \mathrm{~km}$ is the limit below which only a very few $\mathrm{TiO}_{2}$-depleted pyropes occur and pyrope $\mathrm{Mg} \#$ values are less variable. Harzburgitic garnets with depleted $\mathrm{Y}$ and Ti contents exist only at temperatures below $1150^{\circ} \mathrm{C}$; the rare depleted grains from higher temperatures are all lherzolitic varieties (Figs. 5 and 6a). In the middle mantle layer $(110-175 \mathrm{~km})$ there exists a distinctive group of garnets with low $\mathrm{Mg} \#(0.76-0.78)$ that is absent in the other two mantle horizons. These garnets are classified as lherzolitic and wehrlitic varieties according to Schulze (2003) but probably represent melt-metasomatic garnets based on their high $\mathrm{Fe}$ and Ti contents. In Figure 6a they plot among the most Ti-rich garnets, by having $\mathrm{TiO}_{2}$ in the range of $0.5-1.0 \mathrm{wt} \%$. $\mathrm{Y}$ contents of these low $\mathrm{Mg}$ \# garnets are also elevated (>>10 ppm): in Figure 5 they plot with the bulk of the middle temperature garnets. According to the $\mathrm{Zr}$ Y classification by Griffin \& Ryan (1995; Fig. 7) the Kaavi-Kuopio garnet xenocrysts from the middle and the lower mantle layer (lherzolitic, wehrlitic and megacryst varieties) bear evidence of melt metasomatism, which appears to have been a prevalent process in the deeper levels of the upper mantle prior to, or contemporaneous with kimberlite magmatism. A phlogopite (hydrous) metasomatic trend seems to be absent in all xenocrysts (Fig. 7).

\section{Results}

Figure 8 shows C1-chondrite-normalized (McDonough \& Sun, 1995) rare-earth element (REE) profiles of garnets from the Kaavi-Kuopio Province peridotite xenoliths (Fig. 8a; Peltonen et al., 1999) and selected garnet xenocrysts (Figs. 8b-8g). Based on 

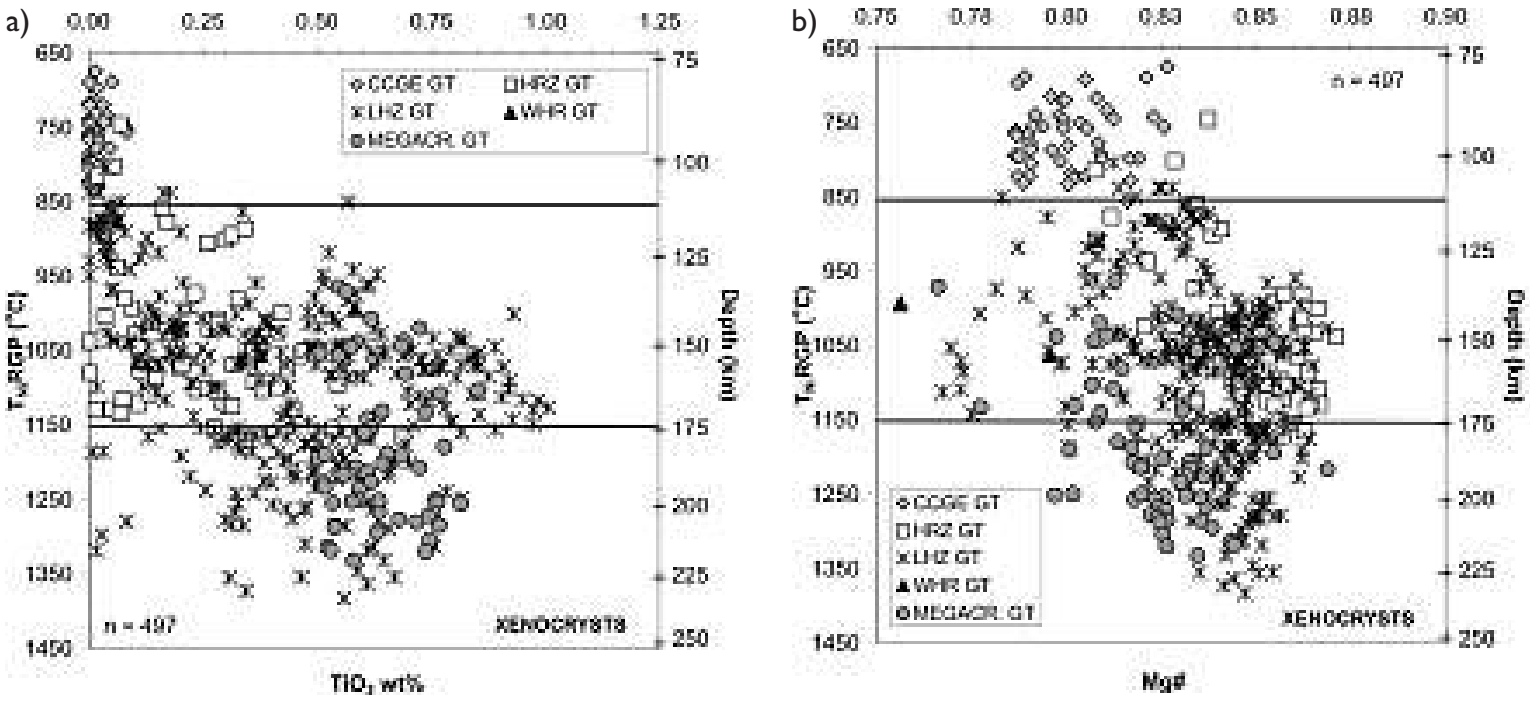

Fig. 6. $\mathrm{TiO}_{2}$ a) and $\mathrm{Mg \#} \mathrm{b)} \mathrm{vs.} \mathrm{T}_{\mathrm{Ni}}$ (Ryan et al., 1996) and depth for the Kaavi-Kuopio xenocryst pyropes showing three distinct layers of the underlying mantle. Depths are calculated as in Fig. 5. Cr-rich $\left(\mathrm{Cr}_{2} \mathrm{O}_{3}>1.5 \mathrm{wt} \%\right)$ megacryst composition grains are included in the diagrams, see Fig. 4.

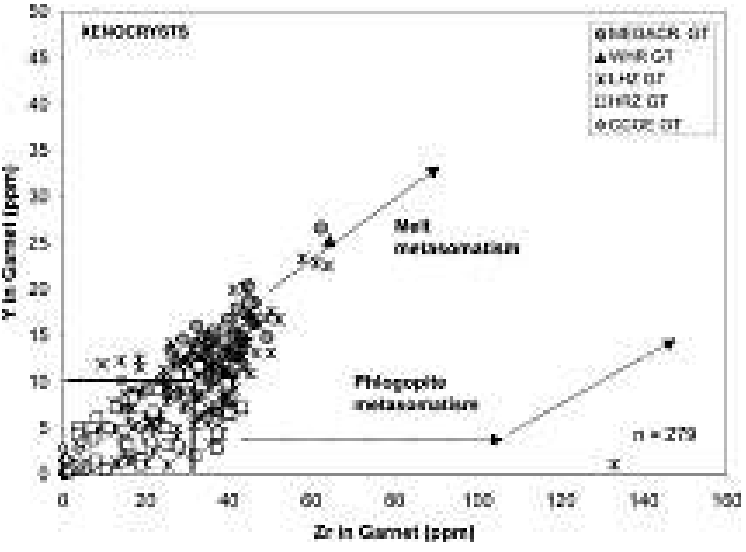

Fig. 7. Y vs. Zr for the Kaavi-Kuopio pyrope xenocrysts (from Lehtonen et al., 2004). Melt and phlogopite metasomatism trends are drawn after Griffin and Ryan (1999b). Solid black line defines the depleted field.

major and trace element compositions and $\mathrm{Ni}$ thermometry results described in section 4 , the garnet xenocrysts can be classified into six classes, as listed in Table 3. These are discussed further below.

\section{I. Rare-earth element contents of garnets in peridotite xenoliths}

The $\mathrm{REE}_{\mathrm{N}}$ patterns of garnets in the garnet facies peridotite xenoliths (Fig. 8a) are very similar regardless of their host rock type (lherzolite, harzburgite or wehrlite defined by modal petrography), showing strong LREE depletion relative to MREE and HREE with steady enrichment from $\mathrm{Sm}_{\mathrm{N}}$ to $\mathrm{Yb}_{\mathrm{N}}$, typical of Casaturated mantle garnets (e.g. Shimizu, 1975). This type of garnet $\mathrm{REE}_{\mathrm{N}}$ patterns are herein termed "Ntype". Garnets from garnet-spinel facies harzburgite xenoliths have ultra-depleted $\mathrm{REE}_{\mathrm{N}}$ profiles, particularly in terms of MREE and HREE. Garnet analyzed from a phlogopite- and Cpx-bearing garnet-spinel symplectite in L14 xenolith has a convex-upward $\mathrm{REE}_{\mathrm{N}}$ pattern that shows strong MREE (Nd to Eu) enrichment followed by a decrease towards HREE. The symplectites have been taken to represent decomposed primary garnet (Peltonen et al., 1999).

\subsection{Rare-earth element contents of peridotitic garnet xenocrysts}

The $\mathrm{REE}_{\mathrm{N}}$ profiles of all garnet xenocrysts classified as megacrysts (Table 3, Class 6; Fig. 8g) and most of the xenocrysts classified as intermediate temperature (Class 4; Fig. 8e) to high temperature (Class 5; Fig. 8f) lherzolitic pyropes are N-type, similar to those from the garnet facies peridotite xenoliths described above. A subset of the Ti- and $\mathrm{Y} \pm \mathrm{Zr}$ poor 
a)

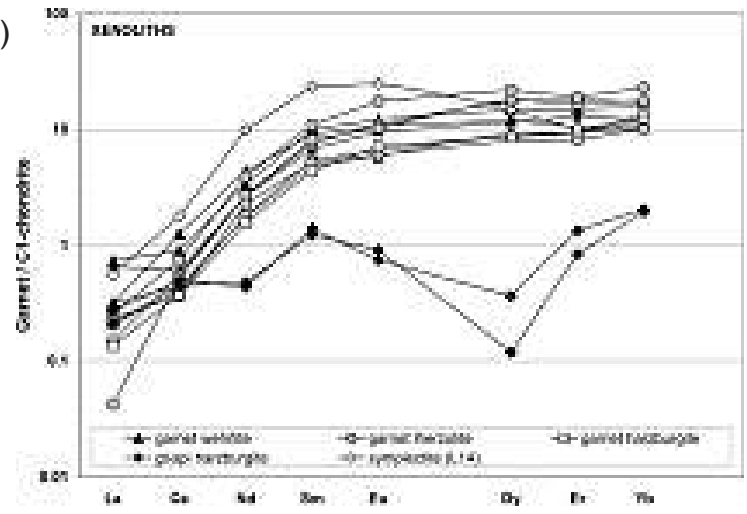

b)

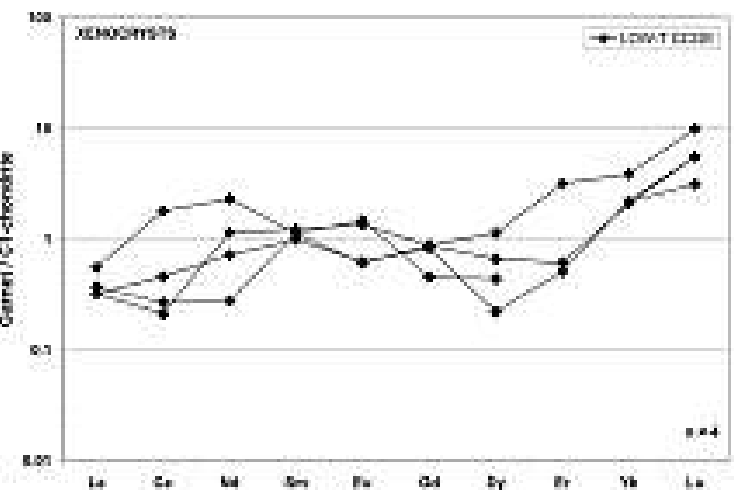

c)

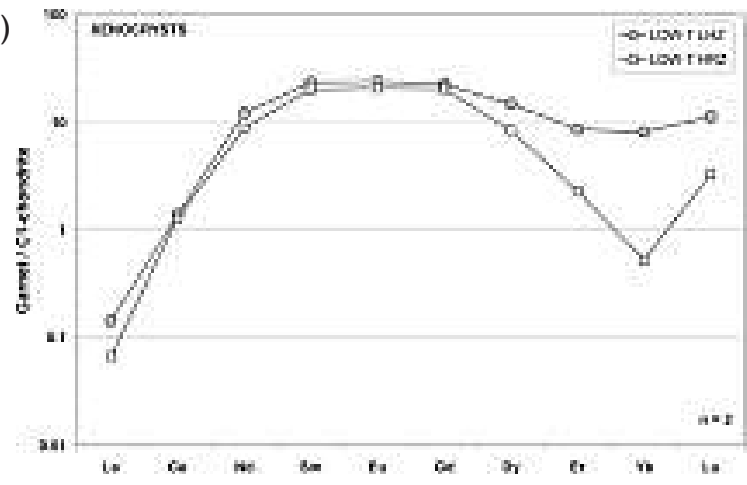

d)

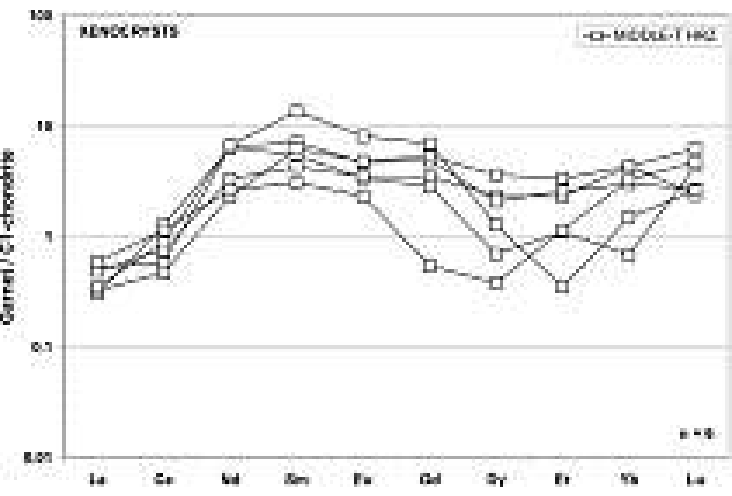

e)
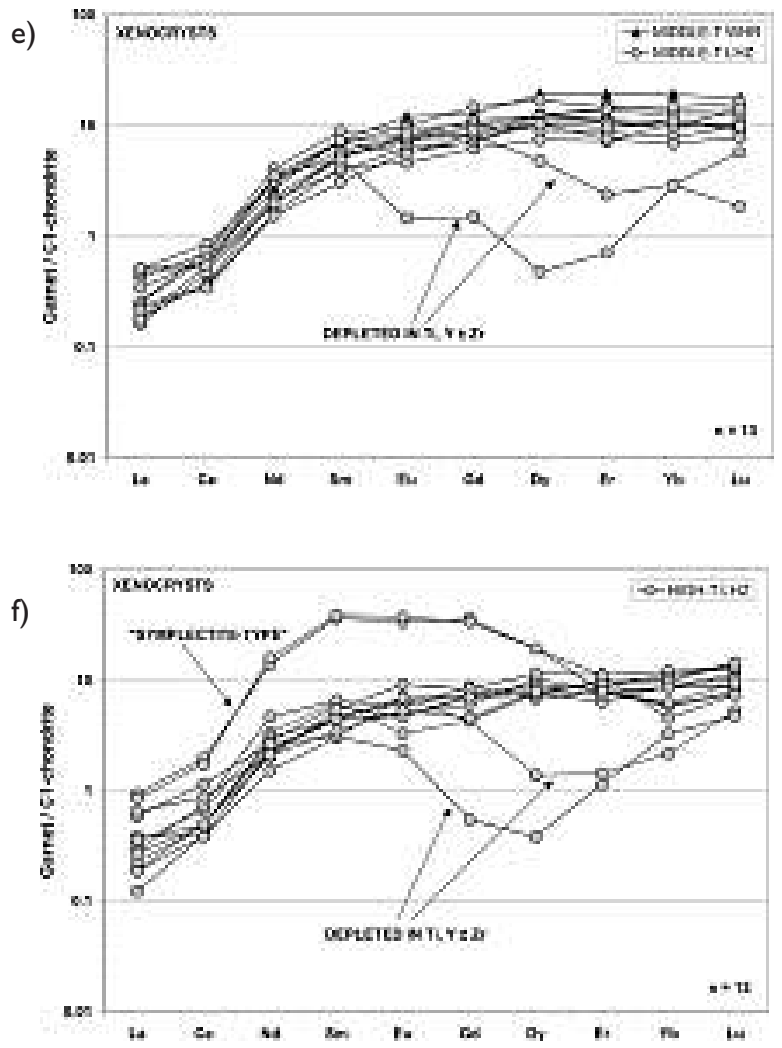

g)

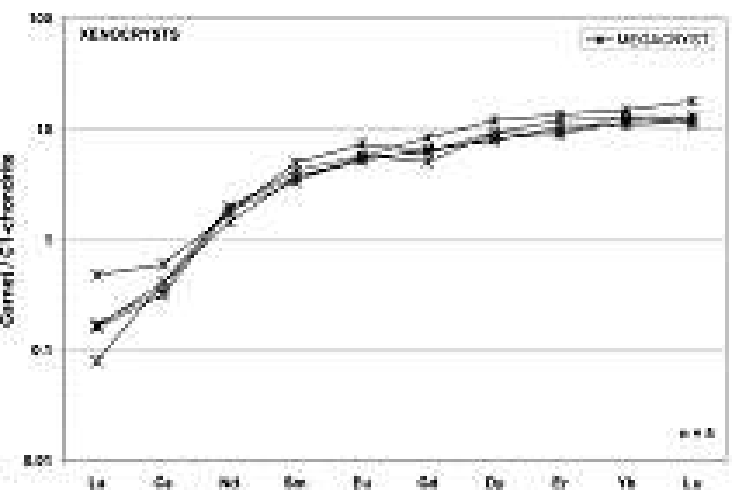

Fig. 8. Cl-Chondrite normalized (McDonough \& Sun, 1995) REE profiles of garnets from (a) xenoliths and (b-g) of garnet xenocrysts. Gd and Lu data not available from xenoliths. See text for discussion. 
Table 3. Garnet xenocryst classification based on major and trace element geochemistry and Ni thermometry.

\begin{tabular}{l|l|c|c|c|l}
\hline Class & $\begin{array}{l}\text { Classification } \\
\text { Schulze (2003) }\end{array}$ & $\begin{array}{l}\text { TNi (oC) garnet } \\
\text { Ryan et al. (1996) }\end{array}$ & $\begin{array}{l}\text { Mantle layer } \\
(\mathrm{km})\end{array}$ & Figure & $\begin{array}{l}\text { REE profile normalized to C1-chondrite } \\
\text { (McDonough \& Sun, 1995) }\end{array}$ \\
\hline 1 & CCGE* & $<850$ & $<110$ & $8 \mathrm{~B}$ & ultra-depleted \\
2 & LHZ, HRZ & $<850$ & $<110$ & $8 \mathrm{C}$ & convex upward (i.e. symplectite-type) \\
3 & HRZ & $850-1150$ & $110-175$ & $8 \mathrm{D}$ & sinusoidal \\
4 & LHZ, WHR & $850-1150$ & $110-175$ & $8 \mathrm{E}$ & N-type, rare sinusoidal \\
5 & LHZ & $>1150$ & $>175$ & $8 \mathrm{~F}$ & N-type, very rare sinusoidal or symplectite-type \\
6 & MEGACR. & mostly $>1150$ & mostly $>175$ & $8 \mathrm{G}$ & N-type \\
\hline
\end{tabular}

*) Classification after Kopylova et al. (2000) for distinctive Ca-saturated and Ti-, Y and Zr-depleted pyropes.

Classified as lherzolitic pyropes according to Schulze (2003).

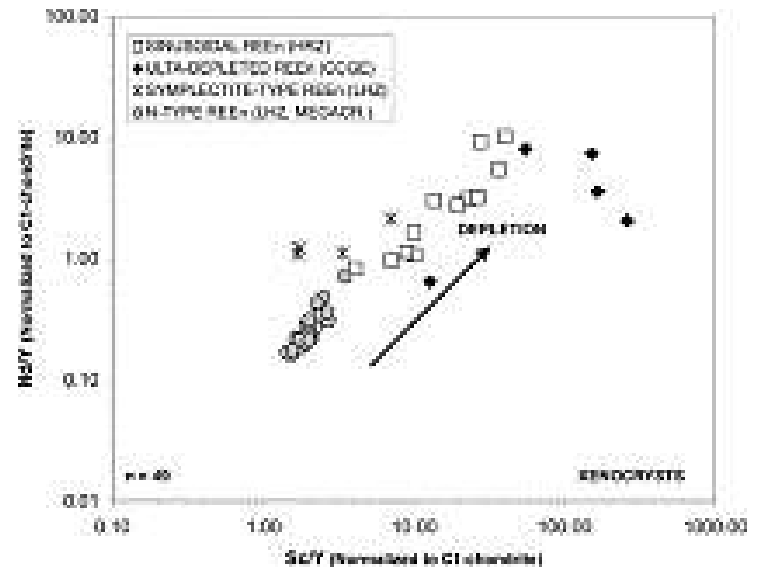

Fig. 9. $\mathrm{Sc} / \mathrm{Y}(\mathrm{N})$ vs. $\mathrm{Nd} / \mathrm{Y}(\mathrm{N})$ for garnet xenocrysts. $(\mathrm{N}$ = normalized to $\mathrm{Cl}$-chondrite composition after McDonough \& Sun, 1995).

$\left(\mathrm{TiO}_{2}<0.4 \mathrm{wt} \% ; \mathrm{Y} \pm \mathrm{Zr}<10 \mathrm{ppm}\right)$ lherzolitic and nearly all of the intermediate temperature harzburgitic (Class 3; Fig. 8d) grains have sinusoidal $\mathrm{REE}_{\mathrm{N}}$ patterns, diagnostic for subcalcic harzburgitic garnets (e.g. Shimizu, 1975). The positive slope from $\mathrm{La}_{\mathrm{N}}$ to $\mathrm{Nd}_{\mathrm{N}}$ is due to a strong increase in compatibility in the garnet structure with decreasing ionic radius of the LREE from La to Nd (e.g. Stachel et al., 1998). The sinusoidal $\mathrm{REE}_{\mathrm{N}}$ profile is characterized by enrichment in Sm over Dy, and LREE enrichment, as measured, for instance by $(\mathrm{Nd} / \mathrm{Y})_{\mathrm{N}}$ greater than unity (Fig. 9). The $(\mathrm{Nd} / \mathrm{Y})_{\mathrm{N}}$, in fact, distinguishes garnets with sinusoidal $\mathrm{REE}_{\mathrm{N}}$ patterns and $(\mathrm{Nd} / \mathrm{Y})_{\mathrm{N}}>>1$, from those with LREE depleted N-type patterns and $(\mathrm{Nd} / \mathrm{Y})_{\mathrm{N}}<<1$ (Pearson et al., 1998). The $(\mathrm{Sc} / \mathrm{Y})_{\mathrm{N}}$ ratio of garnets (Fig. 9) provides a measure of the depletion of HREE, since Sc is preferentially accommodat- ed into garnet during depletion. Values of $(\mathrm{Sc} / \mathrm{Y})_{\mathrm{N}}>>1$ are characteristic of depleted compositions, whereas $(\mathrm{Sc} / \mathrm{Y})_{\mathrm{N}} \sim 1$ are obtained from undepleted garnet varieties (Pearson et al., 1998).

The ultra-depleted $\mathrm{REE}_{\mathrm{N}}$ profiles of the low temperature CCGE garnet xenocrysts (Class 1; Fig. 8b) coincide with those from garnets in the garnet-spinel harzburgite xenoliths (Fig. 8a). These $\mathrm{REE}_{\mathrm{N}}$ profiles show a distinct positive slope in HREE from Er/Dy to $\mathrm{Lu}$. This slope has been interpreted to represent the memory of a previous melt depletion event (Stachel et al., 1998). In Figure 9 CCGE garnets are clearly distinct from the other garnet varieties with high $(\mathrm{Sc} /$ $\mathrm{Y})_{\mathrm{N}}$ and $(\mathrm{Nd} / \mathrm{Y})_{\mathrm{N}}$ indicating extreme depletion.

The rare low temperature garnet xenocrysts other than CCGE's, i.e. lherzolitic and harzburgitic grains (Class 2; Fig. 8c), have similar convex-upward $\mathrm{REE}_{\mathrm{N}}$ patterns to garnet analyzed from L14 xenolith (Fig. 8a). Garnet xenocrysts with this type of $\mathrm{REE}_{\mathrm{N}}$ patterns also form a minor portion of the high temperature lherzolitic grains (Class 5, Fig. 8f) but are absent among the intermediate temperature xenocrysts. In Figure 9 these "symplectite-type" garnets partially overlap with the sinusoidal varieties by having $(\mathrm{Nd} / \mathrm{Y})_{\mathrm{N}}$ ratios within the range 1-3 but stand out from them by having lower $(\mathrm{Sc} / \mathrm{Y})_{\mathrm{N}}$ ratios, i.e. closer to unity.

\section{Discussion}

Figure 10 presents the REE profiles of garnets from the peridotite xenoliths and garnet xenocrysts normalized to a garnet from a well-equilibrated hightemperature sheared lherzolite xenolith with primi- 
a)

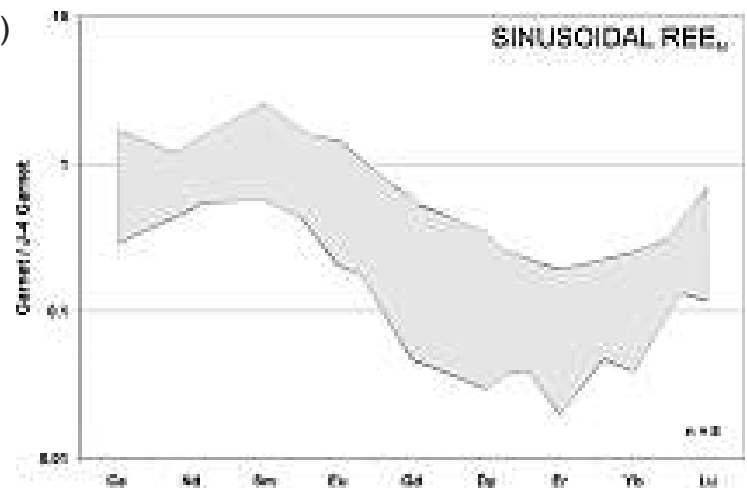

b)

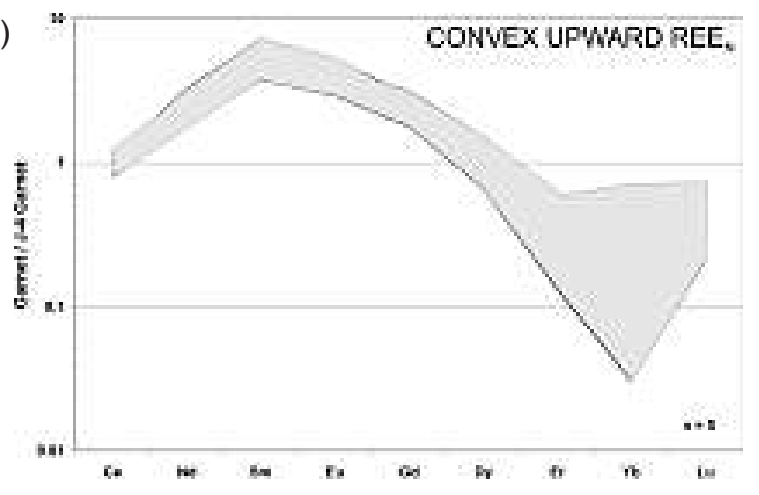

c)

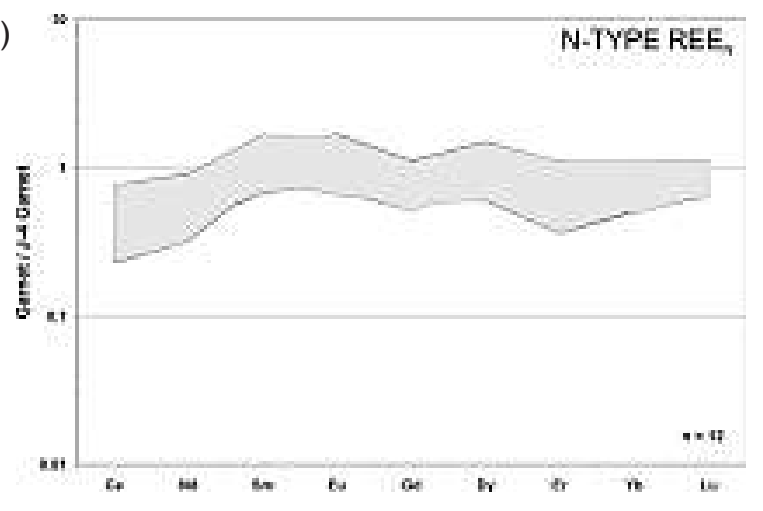

d)

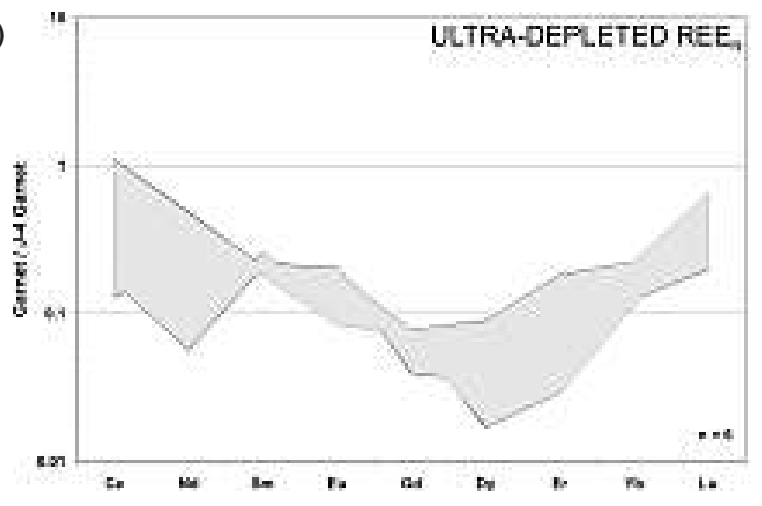

tive, undepleted mantle composition (J-4 from Jagersfontein; Table 1 in Stachel et al., 1998). Normalization to a primitive garnet instead of $\mathrm{C} 1$-chondrite has the advantage that the resulting REE patterns are not dominated by crystal chemical effects, such as the strong increase in compatibility with decreasing ionic radius from LREE to HREE. From the garnet REE contents the following evolutionary history can be suggested for the lithospheric mantle underlying Kaavi-Kuopio.

\section{I. Stage I: Sinusoidal garnet formation}

The sinusoidal $\mathrm{REE}_{\mathrm{N}}$ profiles (Fig. 10a) of the subcalcic harzburgitic (Class 3) and the rare Ti-poor lherzolitic (Classes 4 and 5) garnet xenocrysts indicate that the primitive mantle experienced an extensive komatiite melt extraction event similar to that observed elsewhere, resulting in extreme LREE and HREE depletion, the first step in the formation of cratonic peridotites (e.g. Shimizu \& Richardson, 1987; Nixon et al., 1987; Stachel et al., 1998). Subsequently the lithosphere was most likely composed of highly depleted garnet harzburgite and Ti-poor lherzolite, the residues of this melt extraction process. Sinusoidal $\mathrm{REE}_{\mathrm{N}}$ patterns were developed as a result of fluid metasomatism involving introduction of (repeated) pulses of a fractionated fluid/melt with low HREE and variable LREE/MREE into the depleted lithosphere (Stachel et al., 2004). With respect to their $\mathrm{Y}$ and $\mathrm{Zr}$ contents, the subcalcic harzburgitic garnets plot in the compositional field of depleted garnets (Fig. 7), underlining the highly fractionated

Fig. 10.The four principal types of Kaavi-Kuopio garnets according to their REE patterns normalized to garnet from a well-equilibrated garnet Iherzolite xenolith with primitive mantle composition (J-4 from Jagersfontein, Stachel et al., 1998). a) sinusoidal subcalcic harzburgitic and rare sinusoidal Ti-poor Iherzolitic garnet xenocrysts b) convex upward garnet xenocrysts and a garnet from a symplectite in LI4 xenolith c) garnets from garnet peridotite xenoliths and $\mathrm{N}$-type Iherzolitic and megacryst garnet xenocrysts, and d) ultra-depleted CCGE garnet xenocrysts and garnets from garnet-spinel harzburgite xenoliths. See text for discussion. 
character the fluid/melt must have had. The sinusoidal REE pattern was previously thought to arise from disequilibrium effects, causing the larger LREE cations to diffuse faster than the smaller HREE in garnet structure (Hoal et al., 1994), but experiments do not confirm this process (Ganguly et al., 1998).

Garnets with convex-upward $\mathrm{REE}_{\mathrm{N}}$ patterns, i.e. the symplectite type varieties (Classes 2 and 5), normalized to J4 garnet, have an extreme MREE hump at nearly 10 times primitive garnet and a strong decrease towards HREE (Fig. 10b). They are considered as a subtype of sinusoidal $\mathrm{REE}_{\mathrm{N}}$ garnets described above. According to Ivanic et al. (2003), the very steep positive slope from LREE to MREE of these garnets is likely to have been produced by an evolved metasomatic fluid or melt with $\mathrm{Sm}_{\mathrm{N}} / \mathrm{Lu}_{\mathrm{N}}>>1$ and with $\mathrm{Sm}_{\mathrm{N}} /$ $\mathrm{La}_{\mathrm{N}} \leq 1$. The authors suggest that the formation of such LREE-enriched melts may result from fractional crystallization where garnet itself plays the major role in generating the extreme LREE enrichment over HREE because of its widely varying partition coefficients.

\subsection{Stage 2: $\mathrm{N}$-type garnet formation}

Figure 10c illustrates that the $\mathrm{N}$-type varieties, i.e. the bulk of the xenolith-hosted, lherzolitic and megacryst classes, are closest to the primitive J4 garnet composition in terms of REE contents. Based on subparallel chondrite-normalized whole rock REE patterns and variable $\mathrm{Al}_{2} \mathrm{O}_{3}$ contents of the garnet facies peridotite xenoliths, Peltonen et al. (1999) concluded that these rocks originally formed as ultramafic cumulates from mafic-ultramafic melts with broadly chondritic REE distribution (E-MORB). These cumulates could represent early Proterozoic oceanic lithosphere, tectonically subducted and now underplating the craton margin, and/or younger asthenospheric additions to the base of the lithosphere. Another monitor of mantle fertility is the $\mathrm{Cr}$ content of mantle garnets that is mainly controlled by the level of major element depletion in the host peridotite (Griffin et al., 1999a, 1999b). Indeed, the "primitive" REE profiles of the lherzolitic garnet varieties (Fig. 10c) with con- tradictory depleted major element chemistries (e.g. $\mathrm{Cr}_{2} \mathrm{O}_{3}>>4 \mathrm{wt} \%$ ) are not considered as a primary feature but may have also resulted from metasomatic re-enrichment (Griffin et al., 1989b, Stachel et al., 1998), such as infiltration of the Archean mantle by a metasomatic melt close in composition to megacrystforming magma (Burgess \& Harte, 2004). There are some considerations that favor the idea of re-fertilization in the Kaavi-Kuopio mantle section. The $\mathrm{Y}-\mathrm{Zr}$ classification (Fig. 7) indicates melt metasomatism. The Ti contents of the garnets from the garnet facies peridotite xenoliths and the $\mathrm{N}$-type garnet xenocrysts classified as lherzolitic/wehrlitic types are clearly elevated $\left(\mathrm{TiO}_{2}\right.$ up to $0.89 \mathrm{wt} \%$ and $1.01 \mathrm{wt} \%$, respectively) and, moreover, their REE contents are identical to those of the megacryst composition pyropes (Figs. 8a, e-g). Although few in number, the existence of high-temperature Ti-poor lherzolitic grains with sinusoidal $\mathrm{REE}_{\mathrm{N}}$ profiles (Fig. 8f) is extremely significant for the hypothesis of melt metasomatism. The latter type of garnet xenocrysts possibly originated from remnants of depleted material that was once predominant at depths greater than $180 \mathrm{~km}$ but that was virtually destroyed by re-fertilization as evidenced by the abundance of Ti-rich pyropes. This MREE, HREE and Ti enrichment event did not affect these rare depleted high-T lherzolitic grains nor the medium-T subcalcic harzburgitic (Fig. 8d) and Ti-poor lherzolitic varieties (Fig. 8e). Lower LREE concentrations in lherzolitic garnets compared to harzburgitic grains may be a consequence of the redistribution of REE during re-equilibration with Cpx (Stachel et al., 1998). Wang (1998), for example, has demonstrated that the $\mathrm{Cpx} /$ garnet partition coefficient for $\mathrm{Ce}$ is 2.91 and for $\mathrm{Yb} 0.02$.

\subsection{Stage 3: CCGE garnet formation}

Kopylova et al. (2000) suggested that Ca-saturated Cpx-bearing garnet-spinel cratonic peridotites that host CCGE garnets are restricted to extraordinary mantle segments, since garnet and spinel-bearing mantle is usually too depleted to provide sufficient Cpx for development of the CCGE trend. The rari- 
ty of the trend may also derive from the disequilibrium between phases in cratonic spinel-garnet peridotites (Kopylova et al., 2000; Smith \& Boyd, 1992). In the Kaavi-Kuopio mantle segment CCGE garnets are hosted by garnet-spinel harzburgites containing minor amounts $(<5 \%)$ of Cpx. In the xenoliths CCGE garnets exhibit an irregular and amoeboidal texture that is very different from the rounded appearance of garnets in the garnet facies peridotite xenoliths (Peltonen et al., 1999). The amoeboid texture of the CCGE assemblages is typical of spinel-garnetpyroxene intergrowths, which represent equilibrium textures in low-T peridotite xenoliths (Ionov et al., 1993; Field \& Haggerty, 1994).

The ultra-depletion of CCGE garnets with respect to Ti, Y, Zr and REE (Figs. 6, 7, 10D) combined with their contradictory saturation in $\mathrm{Ca}$, indicate that their host-peridotite has experienced a multi-stage history of depletion and enrichment events. The distinct positive slope of CCGE garnets in HREE from Dy/Er to $\mathrm{Lu}$ (Figs. 8b and 10d) has been interpreted elsewhere to represent a memory of an ancient melt extraction event that affected the primitive mantle source (Stachel et al., 1998). According to Kopylova et al. (2000) CCGE garnets may originate from an ultra-depleted mantle that has been later re-fertilized in Ca by some chemical enrichment process. The excess $\mathrm{Ca}$ has led to the crystallization of secondary Cpx and the transformation of harzburgitic garnets to lherzolitic, similar to that described from mantle garnets from suites in Kimberley, South Africa (Schulze, 1995; Griffin et al., 1999b). Interestingly, the proposed enrichment process has not fertilized the trace element contents of the garnets or affected their REE contents. Moreover, the whole rock analyses of the rare Kaavi-Kuopio garnet-spinel facies peridotite xenoliths show that, in fact, they contain less $\mathrm{CaO}$ than the other peridotitic xenolith varieties, $0.19-0.92 \mathrm{wt} \%$ vs. $0.72-1.55 \mathrm{wt} \%$, respectively (Peltonen et al., 1999).

Peltonen et al. (1999) concluded that the low-T garnet-spinel peridotites could represent harzburgitic residues, i.e., remnants of the reworked Archean lithosphere, metasomatized shortly before or during invasion by a kimberlite-derived melt or fluid complete- ly obscuring their ancient isotopic signature. Carbno \& Canil (2002) suggested based on the Slave craton examples that this enrichment event could have been carbonatite metasomatism, where Cpx forms from Opx as a result of the interaction of carbonate melt with harzburgite (Yaxley et al., 1998). However, neither of these events would likely preserve the ultra-depleted nature of CCGE garnets with respect to trace elements, since the distribution of trace elements among all minerals in a peridotite will control the signature of garnet. Overall, the trace element budget for garnet-bearing peridotites is dominated by both Cpx and garnet (Glaser et al., 1999; Schmidberger \& Francis, 2001). An intriguing possibility is that the shallow $(<110 \mathrm{~km})$ CCGE garnet bearing mantle layer has been somehow affected or modified by events connected to the Svecofennian orogeny, since this layer is not observed further into the Karelian craton based on mantle xenocryst studies from the Kuhmo area kimberlites (O'Brien et al., 2003).

\section{Conclusions}

The REE contents of the Kaavi-Kuopio mantle-derived garnet xenocryst bear evidence of an extensive ancient melt extraction event, similar to observations from lithosphere underlying Archean cratons elsewhere. The highly depleted lithosphere was subsequently affected by fluid metasomatism involving introduction of a fractionated fluid/melt low in HREE giving rise to the sinusoidal $\mathrm{REE}_{\mathrm{N}}$ patterns, characteristic for subcalcic harzburgitic garnets worldwide. Lherzolitic garnets, forming N-type REE patterns, document an enrichment event of MREE and HREE probably by metasomatism by silicate melts that also imprinted a Ti-overprint on most of the pyrope garnets. Harzburgitic and rare lherzolitic garnets have remained unaffected in zones or layers by this metasomatic event. Lower LREE concentrations in lherzolitic garnets compared to harzburgitic varieties may be a function of the redistribution of REE during reequilibration with Cpx.

The results from this study are consistent with the previous work that distinguished three layers in the 
lithospheric mantle underlying Kaavi-Kuopio terrain. The subcalcic harzburgitic garnets forming sinusoidal $\mathrm{REE}_{\mathrm{N}}$ patterns do not occur at temperatures $\left(\mathrm{T}_{\mathrm{N}}\right)$ higher than $1150^{\circ} \mathrm{C}$, i.e. at depths greater than $175 \mathrm{~km}$. Garnets originating from the deepest layer of the lithosphere $(>175 \mathrm{~km})$ are mostly Ti-rich megacryst and lherzolitic garnets, but there are also a few lherzolitic grains depleted in Ti, Y and $\mathrm{Zr}$, and with sinusoidal $\mathrm{REE}_{\mathrm{N}}$. These rare grains, as well as the more frequent depleted grains from the $110-175 \mathrm{~km}$ horizon, assumingly represent depleted mantle material that escaped the melt metasomatic event resulting in the formation of abundant Ti-rich garnets. The probably multi-stage history of the shallowest mantle layer $(<110 \mathrm{~km})$ consisting of garnet-spinel-harzburgite and characterized by CCGE garnets remains an open research topic.

\section{Acknowledgements}

This study was funded by the Geological Survey of Finland and the Academy of Finland. Prof. John Gurney and Dr. Andreas Späth from the University of Cape Town are thanked for the access to the LA-ICP-MS. Dr. Hugh O'Brien and an anonymous reviewer are appreciated for revising the manuscript and providing valuable suggestions for improvements. I am also grateful to the Editor-in-Chief, Dr. Petri Peltonen, for many great ideas. Mr. Bo Johanson and Mr. Lassi Pakkanen from the GTK E-beam Laboratory provided the micro analytical data. The GTK Research Laboratory, Dr. Jukka Marmo (head) and the laboratory personnel, are thanked for the support. The backup of professor Ilmari Haapala from the University of Helsinki is appreciated.

\section{References}

Burgess, S.R. \& Harte, B., 2004. Tracing Lithosphere Evolution through the Analysis of Heterogeneous G9-G10 Garnets in Peridotite Xenoliths, II: REE Chemistry. Journal of Petrology 45, 609-633.

Canil, D., 1991. Experimental evidence for the exsolution of cratonic peridotite from high-temperature harzburgite. Earth and Planetary Science Letters 106, 64-72.

Canil, D., Schulze, D.J., Hall, D., Hearn, B.C. \& Milliken, S.M., 2003. Lithospheric roots beneath western Laurentia: the geochemical signal in mantle garnets. Canadian Journal of Earth Sciences 40, 1027-1051.
Carbno, G.B. \& Canil, D., 2002. Mantle structure beneath the SW Slave Craton, Canada: Constraints from Garnet Geochemistry in the Drybones Bay kimberlite. Journal of Petrology 43, 129-142.

Field, S.W. \& Haggerty, S.E., 1994. Symplectites in upper mantle peridotites: development and implications for the growth of subsolidus garnet, pyroxene and spinel. Contributions to Mineralogy and Petrology 118, 138-156.

Ganguly, J., Tirone, M. \& Hervig, R.L., 1998. Diffusion kinetics of samarium and neodymium in garnet, and a method for determining cooling rates of rocks. Science 281, 805-807.

Glaser, S., Foley, S.F. \& Gunther, D., 1999. Trace element compositions of minerals in garnet and spinel peridotite xenoliths from the Vitim volcanic field, Transbaikalia, eastern Siberia. Lithos 48, 263-285.

Green, T.H., 1980. Island arc and continent-building magmatism - A review of petrogenic models based on experimental petrology and geochemistry. Tectonophysics 63, 367-385.

Grégoire, M., Bell, D.R. \& le Roex, A.P., 2003. Garnet lherzolites from the Kaapvaal Craton (South Africa): Trace element evidence for a metasomatic history. Journal of Petrology 44, 629-657.

Griffin, W.L. \& Ryan, C.G., 1995. Trace elements in indicator minerals: area selection and target evaluation in diamond exploration. In: W.L. Griffin (Ed.), Diamond Exploration: Into the $21^{\text {st }}$ Century. Journal of Geochemical Exploration 53, 311-337.

Griffin, W.L., Cousens, D.R., Ryan, C.G., Sie, S.H. \& Suter, G.F., 1989a. Ni in chrome pyrope garnets: A new geothermometer. Contributions to Mineralogy and Petrology 103, 199-202.

Griffin, W.L., Smith, D., Boyd, F.R., Cousens, D.R., Ryan, C.G., Sie, S.H. \& Suter, G.F., 1989b. Trace element zoning in garnets from sheared mantle xenoliths. Geochimica et Cosmochimica Acta 53, 561-567.

Griffin, W.L., Fisher, N.I., Friedman, J., Ryan, C.G. \& O'Reilly, S.Y., 1999a. Cr-pyrope garnets in the lithospheric mantle. I. Compositional systematics and relations to tectonic setting. Journal of Petrology 40, 679-704.

Griffin, W.L., Shee, S.R., Ryan, C.G., Win, T.T. \& Wyatt, B.A., 1999b. Harzburgite to lherzolite and back again: metasomatic processes in ultramafic xenoliths from the Wesselton kimberlite, Kimberley, South Africa. Contributions to Mineralogy and Petrology 134, 232-250.

Gurney, J.J., 1984. A correlation between garnets and diamonds. In: Glover, J.E. \& Harris, P.G., (eds.) Kimberlite occurrence and origin: A basis for conceptual models in exploration. Geol. Dept. and Univ. Ext., University of Western Australia, Publication 8, 143-166.

Hoal, K.E.O., Hoal, B.G., Erlank, A.J. \& Shimizu, N., 1994. Metasomatism of the mantle lithosphere recorded by rare earth elements in garnets. Earth and Planetary Science Letters 126, 303-313. 
Ionov, D.A., Ashchepkov, I.V., Stosch, H.-G., Witt-Eickschen, G. \& Seck, H.A., 1993. Garnet peridotite xenoliths from the Vitim volcanic field, Baikal region: the nature of the garnet-spinel peridotite transition zone in the continental mantel. Journal of Petrology 34, 11411145 .

Ivanic, T., Harte, B., Burgess, S., Gurney, J., 2003. Factors in the formation of sinuous and humped REE patterns in garnet from mantle harzburgite assemblages. In: Extended abstracts. $8^{\text {th }}$ International Kimberlite Conference, Victoria, BC, Canada, 5pp.

Kontinen, A., Paavola, J. \& Lukkarinen, H., 1992. K-Ar ages of hornblende and biotite from Late Archean rocks of eastern Finland; interpretation and discussion of tectonic implications. Geological Survey of Finland, Bulletin 365, $31 \mathrm{p}$.

Kopylova, M.G., Russell, J.K. \& Cookenboo, H., 1999. Petrology of peridotite and pyroxenite xenoliths from the Jericho kimberlite: Implications for the thermal state of the mantle beneath the Slave Craton, northern Canada. Journal of Petrology 40, 79-104.

Kopylova, M.G., Russell, J.K., Stanley, C. \& Cookenboo, H., 2000. Garnet from Cr- and Ca-saturated mantle: implications for diamond exploration. Journal of Geochemical Exploration 68, 183-199.

Kukkonen, I.T. \& Peltonen, P., 1999. Xenolith controlled geotherm for the central Fennoscandian Shield: Implications for lithosphere-asthenosphere relations. Tectonophysics 304, 301-315.

Kukkonen, I.T., Kinnunen, K.A. \& Peltonen, P., 2003. Mantle xenoliths and thick lithosphere in the Fennoscandian Shield. Physics and Chemistry of the Earth 28, 349-360.

Lehtonen, M.L., O’Brien, H.E., Peltonen, P., Johanson, B.S. \& Pakkanen, L.K., 2004. Layered mantle at the Karelian Craton margin: P-T of mantle xenocrysts and xenoliths from the Kaavi-Kuopio kimberlites, Finland. Lithos 77, 593-608.

Lehtonen, M.L., Pakkanen, L.K. \& Johanson, B.S., 2005. Electron microprobe and LA-ICP-MS analyses of garnet xenocrysts from Kaavi-Kuopio area kimberlites. Geological Survey of Finland, Open file report M 41.2/2005/1.

McDonough, W.F., Sun, S.S., 1995. The composition of the Earth. Chemical Geology 120, 223-253.

Nixon, P.H., van Calsteren, P.W.C., Boyd, F.R. \& Hawkesworth, C.J., 1987. Harzburgites with diamond facies from southern African kimberlites. In: Nixon, P.H. (ed.) Mantle Xenoliths, John Wiley \& Sons Ltd., xxx p.

O’Brien, H.E., Lehtonen, M.L., Spencer, R.G. \& Birnie, A.C., 2003. Lithospheric mantle in eastern Finland: 250 $\mathrm{km} 3 \mathrm{D}$ transect. In: Extended abstracts, $8^{\text {th }}$ International Kimberlite Conference, Victoria, BC, Canada, 5pp.

O’Brien, H.E., Peltonen, P. \& Vartiainen, H., 2005. Kimberlites, carbonatites and alkaline rocks. In: Lehtinen, $\mathrm{M}$. et al. (eds.) Precambrian geology of Finland - Key to the evolution of the Fennoscandian Shield. Elsevier Science B.V., Amsterdam, xxx-yyy
O’Brien, H.E. \& Tyni, M., 1999. Mineralogy and Geochemistry of Kimberlites and Related Rocks from Finland. In: Gurney, J.J. et al., (eds.) Proceedings of the $7^{\text {th }}$ International Kimberlite Conference, Cape Town, South Africa, Vol. 2, 625-636.

O’Neill, H.S.C. \& Wood, B.J., 1979. An experimental study of the iron-magnesium partitioning between garnet and olivine and its calibration as a geothermometer. Contributions to Mineralogy and Petrology 70, 59-70.

Pearson, D.G., 1999. The age of continental roots. Lithos 48, 171-194.

Pearson, N.J., Griffin, W.L., Kaminsky, F.V., van Achterbergh, E. \& O'Reilly, S.Y., 1998. Trace element discrimination of garnet from diamondiferous kimberlites and lamproites. In: Extended abstracts, $7^{\text {th }}$ International Kimberlite Conference, Cape Town, South Africa, 673-675.

Peltonen, P., 1999. Silicification of garnet peridotite xenoliths from the Lahtojoki kimberlite pipe, Finland. In: Gurney, J.J. et al., (eds.) Proceedings of the $7^{\text {th }}$ International Kimberlite Conference, Cape Town, South Africa, Vol. 2, 659-663.

Peltonen, P. \& Mänttäri, I., 2001. An ion microprobe U-Th$\mathrm{Pb}$ study of zircon xenocrysts from the Lahtojoki kimberlite pipe, eastern Finland. Bulletin of the Geological Society of Finland 73, 47-58.

Peltonen, P. \& Brügmann, G., submitted. Origin of layered continental mantle (Karelian craton, Finland): Geochemical and Re-Os isotope constraints. Lithos.

Peltonen, P., Huhma, H., Tyni, M. \& Shimizu, N., 1999. Garnet peridotite xenoliths from kimberlites of Finland: Nature of the continental mantle at an Archean craton - Proterozoic mobile belt transition. In: Gurney, J.J. et al., (eds.) Proceedings of the $7^{\text {th }}$ International Kimberlite Conference, Cape Town, South Africa, Vol. 2, 664-675.

Peltonen, P., Kinnunen, K. A. \& Huhma, H., 2002. Petrology of two diamondiferous eclogite xenoliths from the Lahtojoki kimberlite pipe, eastern Finland. Lithos 63, 151-164.

Robinson, B.W. \& Graham, J., 1992. Advances in Electron Microprobe Trace Element Analysis. Journal of Computer-Assisted Microscopy 4, 263-265.

Ryan, C.G., Griffin, W.L. \& Pearson, N.J., 1996. Garnet geotherms: Pressure-temperature data from $\mathrm{Cr}$-pyrope garnet xenocrysts in volcanic rocks. Journal of Geophysical Research 101, 5611-5625.

Saltzer, R.L., Chatterjee, N. \& Grove, T.L., 2001. The spatial distribution of garnets and pyroxenes in mantle peridotites: Pressure-temperature history of peridotites from the Kaapvaal Craton. Journal of Petrology 42, 22152229.

Schulze, D.J., 1995. Low-Ca garnet harzburgites from Kimberley, South Africa: abundance and bearing on the structure and evolution of the lithosphere. Journal of Geophysical Research 100, 513-526. 
Schulze, D.J., 2003. A classification scheme for mantle-derived garnets in kimberlite: a tool for investigating the mantle and exploring for diamonds. Lithos 71, 195-213.

Schmidberger, S.S. \& Francis, D., 2001. Constraints on the trace element composition of the Archean mantle root beneath Somerset Island, Arctic Canada. Journal of Petrology 42, 1095-1117.

Shimizu, N., 1975. Rare earth elements in garnets and clinopyroxenes from garnet lherzolite nodules in kimberlites. Earth and Planetary Science Letters 25, 26-32.

Shimizu, N. \& Richardson, S.H., 1987. Trace element abundance patterns of garnet inclusions in peridotitesuite diamonds. Geochimica et Cosmochimica Acta 51, 755-758.

Smith, D. \& Boyd, F.R., 1992. Compositional zonation in garnets in peridotite xenoliths. Contributions to Mineralogy and Petrology 112, 134-147.

Sobolev, N.V., Lavrentìev, Yu.G., Pokhilenko, N.P. \& Usova, N.P., 1973. Chrome-rich garnets from the kimberlites of Yakutia and their paragenesis. Contributions to Mineralogy and Petrology 40, 39-52.

Stachel, T., Harris, J.W., Tappert, R. \& Brey, G.P., 2003. Peridotitic diamonds from the Slave and the Kaapvaal cratons - similarities and differences based on a preliminary data set. Lithos 71, 489-503.
Stachel, T., Viljoen, K.S., Brey, G. \& Harris, J.W., 1998. Metasomatic processes in lherzolitic and harzburgitic domains of diamondiferous lithospheric mantle: REE in garnets from xenoliths and inclusions in diamonds. Earth and Planetary Science Letters 159, 1-12.

Stachel, T., Vijoen, K.S., McDade, P. \& Harris, J.W., 2004. Diamondiferous lithospheric roots along the western margin of the Kalahari Craton - the peridotitic inclusion suite in diamonds from Orapa and Jwaneng. Contributions to Mineralogy and Petrology 147, 32-47.

Tyni, M., 1997. Diamond prospecting in Finland - a review. In: Papunen H. (ed.) Mineral deposits: research and exploration, where do they meet? Proceedings of the $4^{\text {th }}$ SGA Meeting, Rotterdam, A.A. Balkema, 789-791.

Wang, W., 1998. Formation of diamond with mineral inclusions of "mixed" eclogite and peridotite paragenesis. Earth and Planetary Science Letters 160, 831-843.

Woodland, A. B.\& Peltonen, P., 1999. Ferric iron contents of garnet and clinopyroxene and estimated oxygen fugacities of peridotite xenoliths from the Eastern Finland Kimberlite Province. In: Gurney, J.J. et al., (eds.) Proceedings of the $7^{\text {th }}$ International Kimberlite Conference, Cape Town, South Africa, Vol. 2, 904-911.

Yaxley, G.M., Green, D.H. \& Kamenetsky, V., 1998. Carbonatite metasomatism in the southeastern Australian lithosphere. Journal of Petrology 39, 1917-1930. 